\title{
Vector Space Framework for Unification of One- and Multidimensional Filter Bank Theory
}

\author{
Tsuhan Chen, Member, IEEE, and P. P. Vaidyanathan, Fellow, IEEE
}

\begin{abstract}
A number of results in filter bank theory can be viewed using vector space notations. This simplifies the proofs of many important results. In this paper, we first introduce the framework of vector space, and then use this framework to derive some known and some new filter bank results as well. For example, the relation among the Hermitian image property, orthonormality, and the perfect reconstruction (PR) property is well-known for the case of one-dimensional (1-D) analysis/synthesis filter banks [1]. We can prove the same result in a more general vector space setting. This vector space framework has the advantage that even the most general filter banks, namely, multidimensional nonuniform filter banks with rational decimation matrices, become a special case. Many results in 1-D filter bank theory are hence extended to the multidimensional case, with some algebraic manipulations of integer matrices. Some examples are: the equivalence of biorthonormality and the PR property, the interchangeability of analysis and synthesis filters, the connection between analysis/synthesis filter banks and synthesis/analysis transmultiplexers, etc. Furthermore, we obtain the subband convolution scheme by starting from the generalized Parseval's relation in vector space. Several theoretical results of wavelet transform can also be derived using this framework. In particular, we derive the wavelet convolution theorem.
\end{abstract}

\section{INTRODUCTION}

$\mathbf{R}$ ECENTLY, analysis/synthesis filter banks have attracted much attention in the area of signal processing. They find applications in subband coding, data compression, transmultiplexing, data encryption, etc. Fig. 1 shows the simplest maximally decimated filter bank, a one-dimensional (1-D) uniformly decimated filter bank. The input signal $x(n)$ is split into $M$ subbands by analysis filters $H_{k}(z)$ and then decimated by $M$ to produce the subband signals $x_{k}(n)$. These subband signals are then processed, e.g., quantized, coded, etc., depending on applications. At the synthesis end, these signals are interpolated by $M$-fold expanders and synthesis filters $F_{k}(z)$ and then summed up to give the reconstructed signal $\hat{x}(n)$. A common requirement in most applications is that, $\hat{x}(n)$ should be as 'close' to $x(n)$ as possible. More specifically, without the intermediate processing on subband signals $x_{k}(n)$, we want $\hat{x}(n)=x(n)$. If this is true, we say

Manuscript received October 21, 1992; revised November 19, 1993. This work was supported in part by the National Science Foundation grant MIP 8919196 and by matching funds from Rockwell Inc., and Tektronix, Inc. The associate editor coordinating the review of this paper and approving it for publication was Prof. Sergio D. Cabrera.

T. Chen was with the Department of Electrical Engineering, Califomia Institute of Technology, Pasadena, CA 91125 USA. He is now with AT\&T Bell Laboratories, Holmdel, NJ 07733-3030 USA.

P. P. Vaidyanathan is with the Department of Electrical Engineering, Califomia Institute of Technology, Pasadena, CA 91125 USA.

IEEE Log Number 9401909.

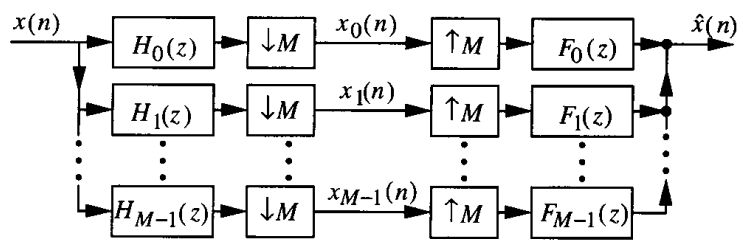

Fig. 1. 1-D uniformly decimated filter bank.

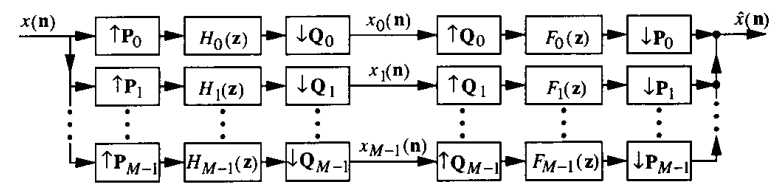

Fig. 2. MD nonuniform rational filter bank.

that this filter bank achieves the perfect reconstruction (PR) property.

The theory and design of 1-D $M$-channel uniform filter banks have been successfully addressed by Vetterli [2], Smith and Barnwell [3], and Vaidyanathan [1]. The extension to nonuniformly decimated filter banks, in which the decimation ratios in each channel are different, has been addressed by Hoang and Vaidyanathan [4], Nayebi et al. [5], and Kovacevic and Vetterli [6]. The extension to the multidimensional (MD) nonuniform case was discussed by Gopinath and Burrus [7]. Fig. 2 shows the most general analysis/synthesis filter bank, namely, an $\mathrm{MD}$ nonuniform filter bank with rational decimation ratios (in this case, decimation matrices). While $\mathbf{P}_{k}$ and $\mathbf{Q}_{k}$ are all nonsingular matrices with integer elements, the equivalent decimation ratio for the $k$ th subband signal is $\mathbf{P}_{k}^{-1} \mathbf{Q}_{k}$, which is a matrix with rational elements. Therefore, this filter bank is said to be an MD nonuniform rational filter bank. This filter bank is said to be maximally decimated if $\sum_{k}\left|\operatorname{det} \mathbf{P}_{k}\right| /\left|\operatorname{det} \mathbf{Q}_{k}\right|=1$. In this case, the sum of sampling rates of subband signals $x_{k}(\mathbf{n})$ is equal to the rate of the input signal $x(\mathbf{n})$.

A number of results in filter bank theory can be viewed using vector space notations. In this paper, we will introduce a vector space framework which incorporates all kinds of filter banks, including discrete wavelet transform (DWT), as special cases. Such a framework simplifies the proofs of many important results. The usefulness of the vector space framework is not surprising in view of the fact that similar interpretations of 1-D filter banks have been made by a number of authors. Among others, these include the vectorial description by Paillard et al. [8], the matrix (possibly infinite- 
dimensional) formulation by Adelson et al. [9], Vetterli and Le Gall [10], the time domain analysis by Nayebi et al. [11], Akansu and Haddad [12], Malvar [13, sec. 4.1.1], and the linear transform formulation by Nuri and Bamberger [14, sec. 3.5.5]. We unify these formulations under the vector space framework, and hence extend many filter bank theoretic results to the most general MD rational filter banks.

\section{A. Paper Outline}

In Section II, we will provide the vector space framework and derive several theorems in this setting. In the following sections, we will apply this framework, with some manipulations of integer matrices, to derive several theoretical results for MD nonuniform rational filter banks which represent the most general case of filter banks. Some of these results are summarized as follows:

1) Consider the following three properties in a maximally decimated filter bank: (a) the Hermitian image property between analysis filters and synthesis filters, i.e. $h_{k}(\mathbf{n})=f_{k}^{*}(-\mathbf{n})$, (b) the orthonormality of the synthesis filters, and (c) the perfect reconstruction (PR) property. For the simplest case of 1-D uniform filter banks, it has been shown that if a filter bank possesses any two of these properties, then it also satisfies the other one [1] It turns out that the same result can be verified in the vector space framework, which covers even the most general MD nonuniform rational filter banks (Section III).

2) A filter bank achieves $P R$ if and only if the analysis filters and synthesis filters are biorthonormal [15], [16]. This result has been informally known but never explicitly shown for the general case of MD nonuniform rational filter banks. In this paper, we will prove it using vector space notations (Section III).

3) The analysis filters and the synthesis filters of a filter bank can be interchanged without changing its PR property (Section III).

4) A synthesis/analysis transmultiplexer [17]-[19] that achieves PR can be obtained by interchanging the analysis part and the synthesis part of a PR maximally decimated analysis/synthesis filter bank. This has been a known result for the 1-D uniform case [19]. Using the vector space framework, we can extend it to the general case (Section IV).

5) $M D$ subband convolution theorem: We shall use the generalized Parseval's relation of vector space to derive the MD subband convolution scheme, for both the orthonormal case (6.8) and the biorthonormal case (6.9). This turns out to be the generalization of the results in [20]. We will also derive the coding gain formula which shows the main advantage of subband convolution (Section VI)

6) Wavelet transform and wavelet convolution theorem: To show further the usefulness of the proposed vector space framework, we will apply it to the discrete wavelet transform [12], [15], [21], [22, ch. 11] to obtain a number of important results. For example, we will show the relation among Hermitian image property, orthonormality, and the PR property, the well-known equivalence of biorthonormality and the PR property, and a new result called the wavelet convolution theorem (7.9) (Section VII).

In Section V, we will justify the linear independence of synthesis filters and their shifts in any PR maximally decimated filter bank, which is required to complete the proofs of results in this paper. To simplify the justification of linear independence, we will present a technique of converting an MD nonuniform rational filter bank into a uniform filter bank with larger number of channels.

\section{B. Notations}

For notations in MD multirate signal processing, refer to [23]-[29], [22, ch. 12]. In particular, an excellent review of MD multirate systems including key notations and concepts is given in [28]. Here, we describe some important notations used in this paper. Capital and lowercase boldfaced letters denote matrices and vectors, respectively. The notation $\left\{\eta_{i}\right\}$ denotes the set of vectors $\eta_{i}$. The symbol $\mathbf{I}_{k}$ represents a $k \times k$ identity matrix (with subscript often omitted). The notations $\mathbf{A}^{T}$ and $\mathbf{A}^{-1}$ denote the transpose and the inverse of $\mathbf{A}$, respectively. The row and column indices typically begin from zero. With $D$ denoting the number of dimensions, $\mathbf{n}=\left[\begin{array}{llll}n_{0} & n_{1} & \cdots & n_{D-1}\end{array}\right]^{T}$ is the 'space' domain index of $\mathrm{MD}$ discrete signals. For example, $x(\mathbf{n})$ represents an image in the 2-D case. Note that all $n_{i}$ are integers. The symbol $\mathcal{N}$ denotes the set of all $D \times 1$ integer vectors, so that $\mathbf{n} \in \mathcal{N}$. The $\mathbf{z}$-transform of $x(\mathbf{n})$ is defined as $X(\mathbf{z})=\sum_{\mathbf{n} \in \mathcal{N}} x(\mathbf{n}) \mathbf{z}^{-\mathbf{n}}$, where $\mathbf{z}=\left[\begin{array}{llll}z_{0} & z_{1} & \cdots & z_{D-1}\end{array}\right]^{T}$ is a complex-valued vector, and a vector raised to a vector power gives a scalar defined as $\mathbf{z}^{-\mathbf{n}} \triangleq z_{0}^{-n_{0}} z_{1}^{-n_{1}} \cdots z_{D-1}^{-n_{D-1}}$.

1) Decimation: The M-fold decimated version of $x(\mathbf{n})$ is defined as $y(\mathbf{n})=x(\mathbf{M n})$, where $\mathbf{M}$ is a nonsingular integer matrix called the decimation matrix. Let $J(\mathbf{M})$ denote $|\operatorname{det} \mathbf{M}|$, the absolute determinant of $\mathbf{M}$. We see that $\mathbf{M}$ fold decimation keeps one sample out of every $J(\mathbf{M})$ input samples.

2) Expansion: For a nonsingular integer matrix $\mathbf{L}$, the $\mathbf{L}$ fold expanded version of $x(\mathbf{n})$ is defined as

$$
y(\mathbf{n})=\left\{\begin{array}{lc}
x\left(\mathbf{L}^{-1} \mathbf{n}\right) & \mathbf{n} \in \operatorname{LAT}(\mathbf{L}) \\
0 & \text { otherwise. }
\end{array}\right.
$$

In the above equation, $\operatorname{LAT}(\mathbf{L})$ (the lattice generated by $\mathbf{L}$ ) denotes the set of all vectors of the form $\mathbf{L m}, \mathbf{m} \in \mathcal{N}$. Clearly, the condition $\mathbf{n} \in \operatorname{LAT}(\mathbf{L})$ above is equivalent to $\mathbf{L}^{-1} \mathbf{n} \in \mathcal{N}$. The matrix $\mathbf{L}$ is called the expansion matrix. The corresponding z-domain relation of expansion is $Y(\mathbf{z})=$ $\mathbf{X}\left(\mathbf{z}^{\mathbf{L}}\right)$. The notation of a vector raised to a matrix power is a $D \times 1$ vector defined as $\mathbf{z}^{\mathbf{L}} \triangleq\left[\begin{array}{llll}\mathbf{z}_{0}^{\ell_{0}} & \mathbf{z}_{1}^{\ell_{1}} & \cdots & \mathbf{z}^{\ell_{D-1}}\end{array}\right]^{T}$, where $\ell_{i}$ is the $i$ th column of $\mathbf{L}$.

\section{ANALYSIS/SYNTHESIS SCHEME IN THE VECTOR SPACE FRAMEWORK}

Instead of dealing with the filter banks directly, we consider a more general setting, viz., vector space (more precisely, inner product space [30]). We will show in later sections the correspondence between vector space notations and filter 
banks. In particular, vectors in the vector space framework will correspond to the input and output signals of filter banks.

Consider a vector space $\mathcal{V}$ defined over a field $\mathcal{F}$. For any two vectors $\mathbf{x}$ and $\mathbf{y}$ in $\mathcal{V}$, we define the inner product, denoted as $(\mathbf{x}, \mathbf{y})$, which gives a scalar in $\mathcal{F}$. The inner product satisfies several properties [30]: For all $\mathbf{x}, \mathbf{y}$, and $\mathbf{z}$ in $\mathcal{V}$ and all $c$ in $\mathcal{F}$, (a) $(\mathbf{x}+\mathbf{z}, \mathbf{y})=(\mathbf{x}, \mathbf{y})+(\mathbf{z}, \mathbf{y})$, (b) $(c \mathbf{x}, \mathbf{y})=c(\mathbf{x}, \mathbf{y})$, (c) $(\mathbf{x}, \mathbf{y})=(\mathbf{y}, \mathbf{x})^{*}$ where the asterisk denotes complex conjugation, and (d) $(\mathbf{x}, \mathbf{x}) \geq 0$, and the equality holds if and only if $\mathbf{x}=\mathbf{0}$. Consider the following analysis/synthesis scheme. Given any vector $\mathbf{x}$ in $\mathcal{V}$, we want to analyze it by computing its inner product with a set of (possibly infinitely many) analysis vectors $\varphi_{i}$ ( $i$ is an integer index)

$$
x_{i}=\left(\mathbf{x}, \varphi_{i}\right) \quad \forall i .
$$

Then, we want to synthesize the vector $\mathrm{x}$ by using $x_{i}$ to form a linear combination of synthesis vectors $\eta_{i}$

$$
\hat{\mathbf{x}}=\sum_{i} x_{i} \eta_{i}
$$

Note that in general $\hat{\mathbf{x}}$ and $\mathbf{x}$ are not identical. Combining the above two equations, we have

$$
\hat{\mathbf{x}}=\sum_{i}\left(\mathbf{x}, \varphi_{i}\right) \eta_{i}
$$

Usually, we want to choose $\varphi_{i}$ and $\eta_{i}$ such that the analysis/synthesis scheme satisfies the perfect reconstruction (PR) condition, i.e., $\hat{\mathbf{x}}=\mathbf{x}$ for all $\mathbf{x}$ in $\mathcal{V}$.

Assumptions on $\eta_{i}$ : For PR to be possible, the span of the set $\left\{\eta_{i}\right\}$ should cover the whole space $\mathcal{V}$, i.e., $\left\{\eta_{i}\right\}$ should be complete. Furthermore, we shall assume that $\eta_{i}$ are linearly independent. Therefore, any vector $\mathbf{x}$ in $\mathcal{V}$ can be expressed as

$$
\mathbf{x}=\sum_{i} a_{i} \eta_{i}
$$

for a unique set of $a_{i}$ in $\mathcal{F}$. These assumptions about completeness and linearly independence will be justified in Section $V$ for the case of filter banks.

In the rest of this section, we shall propose a number of theorems in this vector space framework. These theorems, being easy to prove in vector space notations, will be used to derive many filter bank results in the following sections. The generality of this vector space framework will enable us to derive these results for the 1-D case as well as the MD case, for uniform filter banks as well as nonuniform ones, for the case of rational decimation as well as integer decimation.

Theorem 1: In the above-mentioned analysis/synthesis scheme, any two of the following three conditions imply the other:

a) $\varphi_{i}=\eta_{i}$ for all $i$.

b) $\left(\eta_{i}, \eta_{j}\right)=\delta(i-j)$ for all $i$ and $j$, where $\delta(\cdot)$ is the Kronecker delta, i.e., $\delta(k)$ equals unity for $k=0$ and equals zero otherwise. This is called the orthonormality of the synthesis vectors and the set $\left\{\eta_{i}\right\}$ is said to be an orthonormal set.

c) $\hat{\mathbf{x}}=\mathbf{x}$ for all $\mathbf{x} \in \mathcal{V}$ (perfect reconstruction).
Proof:

1) We first prove that (a) and (b) imply (c). Because we assume that $\left\{\eta_{i}\right\}$ spans $\mathcal{V}$, any $\mathbf{x}$ in $\mathcal{V}$ can be expressed as $\mathbf{x}=\sum_{j} a_{j} \eta_{j}$. Substituting this and (a) into (2.3), we get $\hat{\mathbf{x}}=\sum_{i}\left(\sum_{j} a_{j} \eta_{j}, \eta_{i}\right) \eta_{i}=\sum_{i} \sum_{j} a_{j}\left(\eta_{j}, \eta_{i}\right) \eta_{i}=$ $\sum_{i} \sum_{j} a_{j} \delta(j-i) \eta_{i}=\sum_{i} a_{i} \eta_{i}=\mathbf{x}$. Note that the infinite summation and the inner product can be interchanged, because of the continuity of inner product [30].

2) Next, suppose (a) and (c) are true. Equation (2.3) becomes $\mathbf{x}=\sum_{i}\left(\mathbf{x}, \eta_{i}\right) \eta_{i}, \forall \mathbf{x} \in \mathcal{V}$. Letting $\mathbf{x}=\eta_{j}$, we get $\eta_{j}=\sum_{i}\left(\eta_{j}, \eta_{i}\right) \eta_{i}$. On the other hand, it is clear that $\eta_{j}=\sum_{i} \delta(j-i) \eta_{i}$. Because it is assumed that the expression of any vector in terms of $\eta_{i}$ is unique, we conclude that $\left(\eta_{j}, \eta_{i}\right)=\delta(j-i)$.

3) Finally, suppose (b) and (c) are true. In particular, $\mathbf{x}=\sum_{i}\left(\mathbf{x}, \varphi_{i}\right) \eta_{i}$. The inner product $\left(\mathbf{x}, \eta_{j}\right)=\left(\sum_{i}\left(\mathbf{x}, \varphi_{i}\right) \eta_{i}, \eta_{j}\right)=\sum_{i}\left(\mathbf{x}, \varphi_{i}\right)\left(\eta_{i}, \eta_{j}\right)=$ $\sum_{i}\left(\mathbf{x}, \varphi_{i}\right) \delta(i-j)=\left(\mathbf{x}, \varphi_{j}\right)$, so $\left(\mathbf{x}, \eta_{j}-\varphi_{j}\right)=0$. Letting $\mathbf{x}=\eta_{j}-\varphi_{j}$, we get $\left(\eta_{j}-\varphi_{j}, \eta_{j}-\varphi_{j}\right)=0$ which implies $\eta_{j}=\varphi_{j}$. Note that $\left\{\varphi_{i}\right\}$ is also orthonormal in this case.

$\triangle \triangle \triangle$

Remark: If we do not make the assumption that the set $\left\{\eta_{i}\right\}$ spans $\mathcal{V}$ and $\eta_{i}$ are linearly independent, this theorem should be restated in three parts as follows. If $\left\{\eta_{i}\right\}$ spans $\mathcal{V}$, (a) and (b) imply (c). If $\eta_{i}$ are linearly independent, (a) and (c) imply (b). Finally, (b) and (c) imply (a).

For the same vector space setting, we can relax the orthonormality condition and obtain the following theorem which deals with biorthonormality:

Theorem 2: The analysis/synthesis scheme achieves perfect reconstruction (PR) if and only if $\varphi_{i}$ and $\eta_{i}$ satisfy the biorthonormality condition

$$
\left(\varphi_{i}, \eta_{j}\right)=\delta(i-j) \quad \forall i, j
$$

Proof:

1) If $\mathrm{PR}$ is satisfied, we have $\mathbf{x}=\sum_{i}\left(\mathbf{x}, \varphi_{i}\right) \eta_{i}$. Letting $\mathbf{x}=\eta_{j}$, we get $\eta_{j}=\sum_{i}\left(\eta_{j}, \varphi_{i}\right) \eta_{i}$. On the other hand, $\eta_{j}=\sum_{i} \delta(i-j) \eta_{i}$. We can conclude that $\left(\eta_{j}, \varphi_{i}\right)=$ $\delta(i-j)$.

2) Conversely, suppose (2.5) is true. According to our assumptions, all $\mathbf{x}$ in $\mathcal{V}$ can be expressed as $\mathbf{x}=\sum_{j} a_{j} \eta_{j}$. Substituting this into (2.3), we obtain $\hat{\mathbf{x}}=\sum_{i}\left(\sum_{j} a_{j} \eta_{j}, \varphi_{i}\right) \eta_{i}=\sum_{i} \sum_{j} a_{j}\left(\eta_{j}, \varphi_{i}\right) \eta_{i}=$ $\sum_{i} \sum_{j} a_{j} \delta(i-j) \eta_{i}=\sum_{i} a_{i} \eta_{i}=\mathbf{x} . \quad \Delta \triangle \triangle$

Remark: This theorem should be restated in two parts as follows, if we do not make the assumptions of completeness and linearly independence. If $\eta_{i}$ are linearly independent, then PR implies biorthonormality. If $\left\{\eta_{i}\right\}$ spans $\mathcal{V}$, then the biorthonormality implies PR.

Using Theorem 2, we can verify the following corollary easily:

Corollary 2.1: The vectors $\varphi_{i}$ and $\eta_{i}$ can be interchanged without affecting the PR property of the analysis/synthesis scheme. 
In some applications, e.g., transmultiplexers, the role of the analysis part and the synthesis part is reversed. More specifically, given a set of scalars $x_{i}$ in $\mathcal{F}$, we can synthesize a vector $\mathrm{x}$ using synthesis vectors $\eta_{i}$

$$
\mathbf{x}=\sum_{i} x_{i} \eta_{i}
$$

Then, we can recover $x_{i}$ by analyzing $\mathbf{x}$ using analysis vectors $\varphi_{i}$

$$
\hat{x}_{i}=\left(\mathbf{x}, \varphi_{i}\right) \quad \forall i .
$$

Combining the above two equations, we obtain the following expression for this synthesis/analysis scheme

$$
\hat{x}_{i}=\left(\sum_{j} x_{j} \eta_{j}, \varphi_{i}\right) \quad \forall i
$$

If $\hat{x}_{i}=x_{i}$ for all $i$ and all $x_{i} \in \mathcal{F}$, we say this synthesis/analysis scheme achieves PR. This synthesis/analysis scheme is closely related to the previously mentioned analysis/synthesis scheme, as in the following theorem:

Theorem 3: The analysis/synthesis scheme achieves PR if and only if the corresponding synthesis/analysis scheme achieves PR.

Proof:

1) Suppose the analysis/synthesis scheme achieves $P R$, i.e., $\mathbf{x}=\sum_{i}\left(\mathbf{x}, \varphi_{i}\right) \eta_{i} \quad \forall \mathbf{x} \in \mathcal{V}$. Replacing $\mathbf{x}$ with $\sum_{i} x_{i} \eta_{i}$, we get $\sum_{i} x_{i} \eta_{i}=\sum_{i}\left(\sum_{j} x_{j} \eta_{j}, \varphi_{i}\right) \eta_{i}$. Because $\eta_{i}$ are linearly independent, we conclude that $x_{i}=\left(\sum_{j} x_{j} \eta_{j}, \varphi_{i}\right)$ and therefore the corresponding synthesis/analysis scheme is PR.

2) Conversely, suppose the synthesis/analysis scheme achieves PR, i.e., $x_{i}=\left(\sum_{j} x_{j} \eta_{j}, \varphi_{i}\right) \quad \forall x_{i} \in$ $\mathcal{F}$. Consider the corresponding analysis/synthesis scheme where $\hat{\mathbf{x}}=\sum_{i}\left(\mathbf{x}, \varphi_{i}\right) \eta_{i}$. Because $\left\{\eta_{i}\right\}$ spans $\mathcal{V}$, we can let $\mathbf{x}=\sum_{j} x_{j} \eta_{j}$. So, $\hat{\mathbf{x}}=$ $\sum_{i}\left(\sum_{j} x_{j} \eta_{j}, \varphi_{i}\right) \eta_{i}=\sum_{i} x_{i} \eta_{i}=\mathbf{x}$. That is, the corresponding analysis/synthesis scheme also achieves PR.

$\triangle \triangle \triangle$

Remarks:

1) Without the linear independence and completeness assumptions on the synthesis vectors, this theorem must be restated as follows. If the synthesis vectors $\eta_{i}$ are linearly independent, then a PR analysis/synthesis scheme implies that the corresponding synthesis/analysis scheme achieves PR. If $\left\{\eta_{i}\right\}$ spans $\mathcal{V}$, then a PR synthesis/analysis scheme implies that the corresponding analysis/synthesis scheme is also PR.

2) It is clear from Theorems 2 and 3 that, either for the analysis/synthesis scheme or the synthesis/analysis scheme, biorthonormality is a necessary and sufficient condition for PR.

The following theorem deals with the inner product in the vector space framework:
Theorem 4: Generalized Parseval's relation (biorthonormal case): Suppose $\varphi_{i}$ and $\eta_{i}$ satisfy the biorthonormality condition in (2.5). For any $\mathbf{x}=\sum_{i} x_{i} \eta_{i}$ and any $y=\sum_{i} y_{i} \varphi_{i}$, the inner product of $\mathbf{x}$ and $\mathbf{y}$ can be obtained as follows

$$
(\mathbf{x}, \mathbf{y})=\sum_{i} x_{i} y_{i}^{*}
$$

Proof: Because of (2.5), $(\mathbf{x}, \mathbf{y})=\left(\sum_{i} x_{i} \eta_{i}, \sum_{j} y_{j} \varphi_{j}\right)$ $=\sum_{i} \sum_{j} x_{i} y_{j}^{*}\left(\eta_{i}, \varphi_{j}\right)=\sum_{i} x_{i} y_{i}^{*}$

The following corollary, which applies to the orthonormal case, turns out to be a special case of Theorem 4:

Corollary 4.1: Parseval's relation (orthonormal case): Suppose the set $\left\{\eta_{i}\right\}$ is orthonormal. For any $\mathbf{x}=\sum_{i} x_{i} \eta_{i}$ and any $\mathbf{g}=\sum_{i} g_{i} \eta_{i}$, the inner product of $\mathbf{x}$ and $\mathbf{g}$ can be obtained as follows

$$
(\mathbf{x}, \mathbf{g})=\sum_{i} x_{i} g_{i}^{*}
$$

If we let $\mathbf{g}=\mathbf{x}$, this reduces to the energy conservation equation $(\mathbf{x}, \mathbf{x})=\sum_{i}\left|x_{i}\right|^{2}$.

Furthermore, we can show the following corollary which relates the energy conservation and the orthonormality:

Corollary 4.2: For all $\mathbf{x}$ which can be expressed as $\mathbf{x}=$ $\sum_{i} x_{i} \eta_{i}$, the energy is preserved by $\eta_{i}$, i.e.

$$
(\mathbf{x}, \mathbf{x})=\sum_{i}\left|x_{i}\right|^{2}
$$

if and only if the set $\left\{\eta_{i}\right\}$ is orthonormal.

$\diamond$

Proof: Because the 'if' part is a special case of Corollary 4.1, we only have to prove the 'only if' part. Suppose (2.11) holds for all $\mathbf{x}=\sum_{i} x_{i} \eta_{i}$. Letting $\mathbf{x}=\eta_{i}$ in (2.11), we get $\left(\eta_{i}, \eta_{i}\right)=1$. If we let $\mathbf{x}=a_{i} \eta_{i}+a_{j} \eta_{j}$, where $i \neq j$, we obtain $\left|a_{i}\right|^{2} \underbrace{\left(\eta_{i}, \eta_{i}\right)}_{=1}+\left|a_{j}\right|^{2} \underbrace{\left(\eta_{j}, \eta_{j}\right)}_{=1}+2 \operatorname{Re}\left[a_{i} a_{j}^{*}\left(\eta_{i}, \eta_{j}\right)\right]=\left|a_{i}\right|^{2}+\left|a_{j}\right|^{2}$

where $\operatorname{Re}[\cdot]$ denotes the real part of the argument. So, $\operatorname{Re}\left[a_{i} a_{j}^{*}\left(\eta_{i}, \eta_{j}\right)\right]=0$, for any choice of $a_{i}$ and $a_{j}$. This implies $\left(\eta_{i}, \eta_{j}\right)=0$. Summarizing, we have shown $\left(\eta_{i}, \eta_{j}\right)=\delta(i-j)$, so the set $\left\{\eta_{i}\right\}$ is orthonormal.

$\triangle \triangle \triangle$

\section{Application to Filter BanKS}

In this section, we will apply the vector space framework to the theory of filter banks. We will first consider the simple case of 1-D uniform filter banks (Fig. 1), which most readers are familiar with. Then, we will be ready to extend our discussion to the most general analysis/synthesis filter banks, namely, MD nonuniform filter banks with rational decimation matrices (Fig. 2).

\section{A. 1-D Uniform Filter Banks (the Simplest Case)}

Let us start with the 1-D uniform filter bank shown in Fig. 1. We can express the subband signals as follows

$$
x_{k}(m)=\sum_{n=-\infty}^{\infty} x(n) h_{k}(M m-n), \quad k=0, \cdots, M-1 .
$$


Note that we have changed the time domain index of the subband signals from $n$ to $m$ for convenience in later discussion. Defining $\phi_{k, m}(n) \fallingdotseq h_{k}^{*}(M m-n)$, we can rewrite (3.1) as

$$
x_{k}(m)=\sum_{n=-\infty}^{\infty} x(n) \phi_{k, m}^{*}(n), \quad k=0, \cdots, M-1
$$

Similarly, we can express the reconstructed signal $\widehat{x}(n)$ as

$$
\widehat{x}(n)=\sum_{k=0}^{M-1} \sum_{m=-\infty}^{\infty} x_{k}(m) f_{k}(n-M m) .
$$

Define $\eta_{k, m}(n) \triangleq f_{k}(n-M m)$. The above equation can be rewritten as

$$
\widehat{x}(n)=\sum_{k=0}^{M-1} \sum_{m=-\infty}^{\infty} x_{k}(m) \eta_{k, m}(n) .
$$

We say this system achieves perfect reconstruction (PR) if $\widehat{x}(n)=x(n)$ for all $x(n)$.

Such an analysis/synthesis filter bank is essentially a special case of the vector space analysis/synthesis framework mentioned in Section II. To see this, consider the vector space $\mathcal{V}$ which is the set of all sequences with finite energy, i.e., all $x(n)$ such that $\sum_{n=-\infty}^{\infty}|x(n)|^{2}$ is finite (i.e., the $\ell_{2}$ space [30]). With $\mathcal{F}$ being the field of complex numbers, we define the inner product of two signals in $\ell_{2}$ as

$$
(x(n), y(n)) \triangleq \sum_{n=-\infty}^{\infty} x(n) y^{*}(n) .
$$

It can be verified that this definition satisfies all the fundamental properties of inner product. Note that we use the $\ell_{2}$ space here so that the inner product defined above always exists. Consider the following correspondences between sequences and vectors in the vector space framework

$$
\begin{aligned}
x(n) & \longleftrightarrow \mathbf{x} \\
\phi_{k, m}(n) & \longleftrightarrow \varphi_{i} \\
x_{k}(m) & \longleftrightarrow x_{i} .
\end{aligned}
$$

Note that the time index $n$ has been absorbed in the vector notations, and the indices $k$ and $m$ are combined and rearranged to become the single index $i$. That is, for any pair of $k$ and $m$, there exists a unique $i$. Using (3.5) and (3.6), we can rewrite (3.2) in vector space notations and obtain $x_{i}=\left(\mathbf{x}, \varphi_{i}\right)$ which is exactly (2.1), the analysis part in the vector space framework. Similarly, using

$$
\begin{aligned}
\eta_{k, m}(n) & \longleftrightarrow \eta_{i} \\
\widehat{x}(n) & \longleftrightarrow \widehat{\mathbf{x}}
\end{aligned}
$$

we can rewrite (3.4) as $\widehat{\mathbf{x}}=\sum_{i} x_{i} \eta_{i}$ which is exactly (2.2), the synthesis part in the vector space framework. Therefore, we have shown that the 1-D uniform filter bank is indeed a special case of the vector space framework mentioned in Section II.

\section{B. MD Nonuniform Rational Filter Banks (the Most General Case)}

We now proceed to Fig. 2 which shows the most general $M$-channel analysis/synthesis filter bank, namely, an MD nonuniform filter bank with rational decimation matrices. As we will show, this is also covered as a special case by the proposed framework. Here, we can assume that $\mathbf{P}_{k}$ and $\mathbf{Q}_{k}$ are left coprime [31]-[33] for all $k$, because any common left factor can be canceled in $\mathbf{P}_{k}^{-1} \mathbf{Q}_{k}$ without affecting the decimation ratio in that channel. Also assume that $\sum_{k} J\left(\mathbf{P}_{k}\right) / J\left(\mathbf{Q}_{k}\right)=1$ for maximal decimation.

Using the definitions of decimation and expansion, we can express the subband signal $x_{k}(\mathbf{n})$ as follows

$x_{k}(\mathbf{m})=\sum_{\mathbf{n} \in \mathcal{N}} x(\mathbf{n}) h_{k}\left(\mathbf{Q}_{k} \mathbf{m}-\mathbf{P}_{k} \mathbf{n}\right), \quad k=0, \cdots, M-1$.

Again, we have changed the space domain index $\mathbf{n}$ of the subband signals to $\mathbf{m}$. Defining $\phi_{k \mathbf{m}}(\mathbf{n}) \triangleq h_{k}^{*}\left(\mathbf{Q}_{k} \mathbf{m}-\mathbf{P}_{k} \mathbf{n}\right)$, we rewrite the above as

$$
x_{k}(\mathbf{m})=\sum_{\mathbf{n} \in \mathcal{N}} x(\mathbf{n}) \phi_{k \mathbf{m}}^{*}(\mathbf{n}), \quad k=0, \cdots, M-1 .
$$

On the other hand, the reconstructed signal $\widehat{x}(\mathbf{n})$ can be expressed as

$$
\widehat{x}(\mathbf{n})=\sum_{k=0}^{M-1} \sum_{\mathbf{m} \in \mathcal{N}} x_{k}(\mathbf{m}) f_{k}\left(\mathbf{P}_{k} \mathbf{n}-\mathbf{Q}_{k} \mathbf{m}\right) .
$$

Define $\eta_{k \mathbf{m}}(\mathbf{n}) \triangleq f_{k}\left(\mathbf{P}_{k} \mathbf{n}-\mathbf{Q}_{k} \mathbf{m}\right)$. These are the $\mathbf{P}_{k}$-fold decimated versions of synthesis filters and their $\mathbf{Q}_{k}$-shifts. The above equation is then rewritten as

$$
\widehat{x}(\mathbf{n})=\sum_{k=0}^{M-1} \sum_{\mathbf{m} \in \mathcal{N}} x_{k}(\mathbf{m}) \eta_{k \mathbf{m}}(\mathbf{n}) .
$$

Remark: If $\widehat{x}(\mathbf{n})=x(\mathbf{n})$ for all $x(\mathbf{n})$, we say this system achieves PR. It can be shown that PR can be achieved by filter banks with ideal filters. However, PR is not always possible for practical filters, i.e., filters having rational transfer functions. Even for the 1-D case with $P_{k}=1$ (nonuniform filter bank with integer decimation ratios), it has been shown that some choices of $Q_{k}$ will make PR impossible for practical filters [4]. Interested readers can read [4] for the idea of compatible sets of $Q_{k}$. Even for the 1-D case, finding a set of necessary and sufficient conditions on $Q_{k}$ such that $\mathrm{PR}$ is possible still remains an open problem. We will not address this issue in this paper. Instead, we will assume that for our discussion, all $\mathbf{Q}_{k}$ are chosen properly such that $\mathrm{PR}$ is indeed possible.

To see that this filter bank is indeed a special case of the vector space analysis/synthesis scheme, consider the $\ell_{2}$ space which is the set of all MD signals with finite energy, i.e., all $x(\mathbf{n})$ such that $\sum_{\mathbf{n} \in \mathcal{N}}|x(\mathbf{n})|^{2}$ is finite. Similar to the 1-D case, we define the inner product of two MD signals as

$$
(x(\mathbf{n}), y(\mathbf{n})) \triangleq \sum_{\mathbf{n} \in \mathcal{N}} x(\mathbf{n}) y^{*}(\mathbf{n}) .
$$

Rearranging the indices $k=0, \cdots, M-1$ and $\mathbf{m} \in \mathcal{N}$ as a single index $i$ in the vector space framework, we obtain the 
following correspondences between MD signals and vectors

$$
\begin{aligned}
x(\mathbf{n}) & \longleftrightarrow \mathbf{x} \\
\phi_{k \mathbf{m}}(\mathbf{n}) & \longleftrightarrow \varphi_{i} \\
x_{k}(\mathbf{m}) & \longleftrightarrow x_{i} \\
\eta_{k \mathbf{m}}(\mathbf{n}) & \longleftrightarrow \eta_{i} \\
\widehat{x}(\mathbf{n}) & \longleftrightarrow \widehat{\mathbf{x}} .
\end{aligned}
$$

From (3.12) and (3.13), we see that (3.9) is essentially $x_{i}=$ $\left(\mathbf{x}, \varphi_{i}\right)$ and (3.11) is $\widehat{\mathbf{x}}=\sum_{i} x_{i} \eta_{i}$. Hence we conclude that an MD nonuniform rational filter bank is indeed a special case of the proposed vector space framework.

Assumptions on $f_{k}\left(\mathbf{P}_{k} \mathbf{n}-\mathbf{Q}_{k} \mathbf{m}\right)$ : The only detail missing here is the assumptions that $\eta_{k \mathbf{m}}(\mathbf{n})$, or $f_{k}\left(\mathbf{P}_{k} \mathbf{n}-\mathbf{Q}_{k} \mathbf{m}\right)$, are linearly independent and the set $\left\{\eta_{k \mathbf{m}}(\mathbf{n})\right\}$ spans the $\ell_{2}$ space. The following theorems hold only under these assumptions, which will be justified in Section V. The readers may notice that the condition of maximal decimation, $\sum_{k} J\left(\mathbf{P}_{k}\right) / J\left(\mathbf{Q}_{k}\right)=1$, has not been explicitly used so far. It turns out that maximal decimation is indeed what makes $\eta_{k \mathbf{m}}(\mathbf{n})$ linearly independent (Section V).

Now, we can prove the following theorem:

Theorem 5: For an MD nonuniform maximally decimated filter bank as shown in Fig. 2, any two of the following conditions imply the other:

a) The Hermitian image property

$$
h_{k}(\mathbf{n})=f_{k}^{*}(-\mathbf{n}) \quad \text { for } k=0, \cdots, M-1 .
$$

b) The orthonormality of the synthesis filters

$$
\begin{aligned}
\sum_{\mathbf{n} \in \mathcal{N}} \underbrace{f_{k}\left(\mathbf{P}_{k} \mathbf{n}-\mathbf{Q}_{k} \mathbf{m}\right)}_{\eta_{k \mathbf{m}}(\mathbf{n})} \underbrace{f_{k^{\prime}}^{*}\left(\mathbf{P}_{k^{\prime}} \mathbf{n}-\mathbf{Q}_{k^{\prime}} \mathbf{m}^{\prime}\right)}_{\begin{array}{c}
\eta_{k^{\prime} \mathbf{m}^{\prime}}^{*}(\mathbf{n}) \\
=\delta\left(k-k^{\prime}\right) \delta\left(\mathbf{m}-\mathbf{m}^{\prime}\right)
\end{array}} \\
\end{aligned}
$$

for all $k, k^{\prime}=0, \cdots, M-1$, and for all $\mathbf{m}, \mathbf{m}^{\prime} \in \mathcal{N}$. Here the Kronecker delta function $\delta(\mathbf{k})$ is extended to have a vector argument, and equals unity when $\mathbf{k}=\mathbf{0}$ and equals zero otherwise.

c) The perfect reconstruction (PR) property

$$
\widehat{x}(\mathbf{n})=x(\mathbf{n}), \quad \text { for all } x(\mathbf{n}) \text { with finite energy. }
$$

Proof: ${ }^{1}$ It turns out that these three conditions all correspond nicely to the three conditions in Theorem 1. For (b) and (c) this fact can be easily seen, because (3.15) can be written as $\left(\eta_{i}, \eta_{i^{\prime}}\right)=\delta\left(i-i^{\prime}\right)$ and (3.16) as $\widehat{\mathbf{x}}=\mathbf{x}$ in vector space notations. Also, the equivalence of Condition (a) in Theorem 1 to Condition (a) in Theorem 5 can be proved as follows

$$
\begin{aligned}
& \varphi_{i}=\eta_{i} \quad \forall i \\
\Longleftrightarrow & \phi_{k \mathbf{m}}(\mathbf{n})=\eta_{k \mathbf{m}}(\mathbf{n}) \quad k=0, \cdots, M-1, \mathbf{m} \in \mathcal{N} \\
\Longleftrightarrow & h_{k}^{*}\left(\mathbf{Q}_{k} \mathbf{m}-\mathbf{P}_{k} \mathbf{n}\right)=f_{k}\left(\mathbf{P}_{k} \mathbf{n}-\mathbf{Q}_{k} \mathbf{m}\right) \\
& k=0, \cdots, M-1, \mathbf{m} \in \mathcal{N} \\
\Longleftrightarrow & h_{k}^{*}(\mathbf{n})=f_{k}(-\mathbf{n}) \quad k=0, \cdots, M-1 .
\end{aligned}
$$

${ }^{1}$ For the special case of 1-D nonuniform filter banks with integer decimation ratios, alternative proofs of Theorem 5 and Theorem 6 have been obtained by Igor Djoković, graduate student, Caltech.

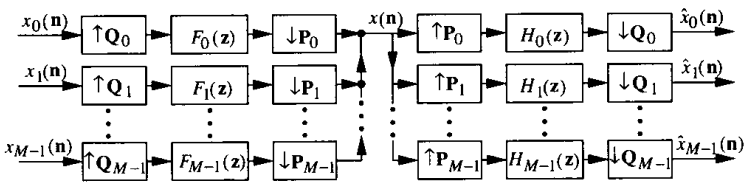

Fig. 3. MD transmultiplexer.

The last equivalence is due to the fact that $\mathbf{P}_{k}$ and $\mathbf{Q}_{k}$ are left coprime (see Appendix A for details). Hence this theorem is indeed a special case of Theorem 1 , and the same relation holds.

$\triangle \triangle \triangle$

We also have the following theorem for the biorthonormal case, which is a direct result of Theorem 2:

Theorem 6: The MD nonuniform maximally decimated filter bank in Fig. 2 satisfies the PR condition if and only if the filters satisfy the biorthonormal property

$$
\begin{aligned}
\sum_{\mathbf{n} \in \mathcal{N}^{\prime}} h_{k}\left(\mathbf{Q}_{k} \mathbf{m}-\mathbf{P}_{k} \mathbf{n}\right) f_{k^{\prime}}\left(\mathbf{P}_{k^{\prime}} \mathbf{n}-\mathbf{Q}_{k^{\prime}} \mathbf{m}^{\prime}\right) & \\
= & \delta\left(k-k^{\prime}\right) \delta\left(\mathbf{m}-\mathbf{m}^{\prime}\right)
\end{aligned}
$$

for all $k, k^{\prime}=0, \cdots, M-1$, and for all $\mathbf{m}, \mathbf{m}^{\prime} \in \mathcal{N}$. $\diamond$

In (3.18), we can replace $\mathbf{n}$ with $-\mathbf{n}, \mathbf{m}$ with $-\mathbf{m}$, and $\mathbf{m}^{\prime}$ with $-\mathbf{m}^{\prime}$, to obtain

$$
\begin{aligned}
\sum_{\mathbf{n} \in \mathcal{N}} f_{k^{\prime}}\left(\mathbf{Q}_{k^{\prime}} \mathbf{m}^{\prime}-\mathbf{P}_{k^{\prime}} \mathbf{n}\right) h_{k}\left(\mathbf{P}_{k} \mathbf{n}-\mathbf{Q}_{k} \mathbf{m}\right) & \\
= & \delta\left(k-k^{\prime}\right) \delta\left(\mathbf{m}-\mathbf{m}^{\prime}\right) .
\end{aligned}
$$

This gives us the following corollary (which is also a direct result of Corollary 2.1):

Corollary 6.1: We can interchange the analysis filters and synthesis filters in any PR maximally decimated filter bank to obtain another PR maximally decimated filter bank.

\section{FILTER BANKS AND TRANSMULTIPLEXERS}

A system which is closely related to filter banks is the so-called transmultiplexer [17]-[19]. A transmultiplexer, as shown in Fig. 3, is obtained by interchanging the analysis part and the synthesis part of an analysis/synthesis filter bank. In this system, input signals $x_{k}(\mathbf{n})$ are processed by $\mathbf{Q}_{k}$-fold expanders, filters $F_{k}(\mathbf{z})$, and $\mathbf{P}_{k}$-fold decimators (equivalent to $\mathbf{P}_{k}^{-1} \mathbf{Q}_{k}$-fold interpolation) and then summed up to form the signal $x(\mathbf{n})$. Effectively, the information in each $x_{k}(\mathbf{n})$ is now carried in a certain frequency band of $x(\mathbf{n})$. Therefore, this is also called the frequency division multiplexing (FDM) technique. Note that $x(\mathbf{n})$ has a higher sampling rate than any of the input signals $x_{k}(\mathbf{n})$. Usually, we choose $\sum_{k} J\left(\mathbf{P}_{k}\right) / J\left(\mathbf{Q}_{k}\right)=1$ such that the sampling rate of $x(\mathbf{n})$ is equal to the sum of the sample rates of $x_{k}(\mathbf{n})$. At the other end, $x(\mathbf{n})$ is separated into $M$ output signals $\widehat{x}_{k}(\mathbf{n})$ by $\mathbf{P}_{k}^{-1} \mathbf{Q}_{k}$-fold decimation with filters $H_{k}(\mathbf{z})$. We want to design the system such that $\widehat{x}_{k}(\mathbf{n})=x_{k}(\mathbf{n})$ for all $k$. When this is true, we say this transmultiplexer has the perfect reconstruction (PR) property.

Starting from Theorem 3 derived in the vector space framework, we can obtain the following theorem which states 
the close relation between analysis/synthesis filter banks and transmultiplexers:

Theorem 7: The MD nonuniform maximally decimated filter bank satisfies the PR condition if and only if the corresponding MD nonuniform transmultiplexer is PR.

This theorem is very useful because it unifies the theory and design of maximally decimated filter banks and those of transmultiplexers. A similar result for the case of 1-D uniform filter banks has been presented in [19]. Here, we have generalized the result therein to the most general case, i.e., the case of MD nonuniform rational filter banks. Also, from Theorems 6 and 7, we see that biorthonormality is necessary and sufficient for the PR property of transmultiplexers as well.

\section{LiNEAR INDEPENDENCE OF $f_{k}\left(\mathbf{P}_{k} \mathbf{n}-\mathbf{Q}_{k} \mathbf{m}\right)$}

When we apply results in the vector space framework to derive Theorems 5-7, we assume that $\eta_{k \mathbf{m}}(\mathbf{n})$, or $f_{k}\left(\mathbf{P}_{k} \mathbf{n}-\right.$ $\left.\mathbf{Q}_{k} \mathbf{m}\right)$, are linearly independent and $\left\{\eta_{k \mathbf{m}}(\mathbf{n})\right\}$ spans the whole space $\ell_{2}$ (completeness). We will now justify these assumptions.

The second assumption about completeness is necessary. If it is not true, there exists an MD signal in $\ell_{2}$ which can not be represented using $\eta_{k \mathbf{m}}(\mathbf{n})$, hence can not be perfectly reconstructed. In this case, PR is out of question, and none of the above theorems is meaningful. So, it only remains to show that $\eta_{k \mathbf{m}}(\mathbf{n})$ are linear independent whenever PR is possible for all $x(\mathbf{n})$. In other words, whenever there exist analysis filters and synthesis filters to achieve PR, the corresponding $\eta_{k \mathbf{m}}(\mathbf{n})$ should be linearly independent. The rest of this section is devoted to show this. The maximal decimation condition, which has not been explicitly used so far, is indeed what makes $\eta_{k \mathbf{m}}(\mathbf{n})$ linearly independent. We will prove this in two steps. First, we will show that $\eta_{k \mathbf{m}}(\mathbf{n})$ are linear independent for a maximally decimated uniform filter bank that has the PR property. We will then show that any MD maximally decimated nonuniform rational filter bank can be converted into a uniform one that has the same set of $\eta_{k \mathbf{m}}(\mathbf{n})$.

\section{A. Linear Independence of $\eta_{k \mathbf{m}}(\mathbf{n})$ for the Uniform Case}

Consider an MD maximally decimated uniform filter bank as shown in Fig. 4(a). We need to show that for this maximally decimated system to achieve PR, the corresponding $\eta_{k \mathbf{m}}(\mathbf{n})=$ $f_{k}(\mathbf{n}-\mathbf{L m})$ are linearly independent. That is, the synthesis filters and their $\mathbf{L}$-shifts are linearly independent. We shall use the MD polyphase decomposition [25], [27], [28] defined as follows:

Polyphase decomposition: The polyphase components of $x(\mathbf{n})$, with respect to a given nonsingular integer matrix $\mathbf{L}$, are defined as

$$
\begin{aligned}
& e_{\mathbf{k}}(\mathbf{n})=x(\mathbf{L n}+\mathbf{k}), \quad(\text { Type 1) } \\
& \text { or } \left.\quad r_{\mathbf{k}}(\mathbf{n})=x(\mathbf{L n}-\mathbf{k}), \quad \text { (Type } 2\right)
\end{aligned}
$$

where $\mathbf{k} \in \mathcal{N}(\mathbf{L})$. Here, $\mathcal{N}(\mathbf{L})$ is the set of all integer vectors of the form $\mathbf{L x}$, where $\mathbf{x}$ is a $D \times 1$ vector with components $x_{i}$ in the range $0 \leq x_{i}<1$. It can be shown that $\mathcal{N}(\mathbf{L})$ contains $J(\mathbf{L})$ distinct vectors, so there are $J(\mathbf{L})$ polyphase

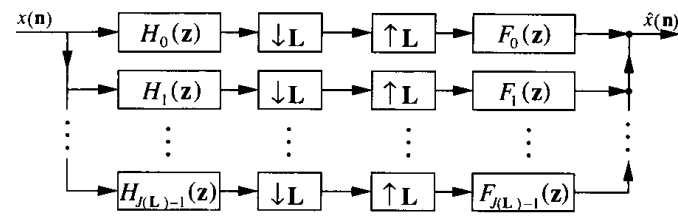

(a)

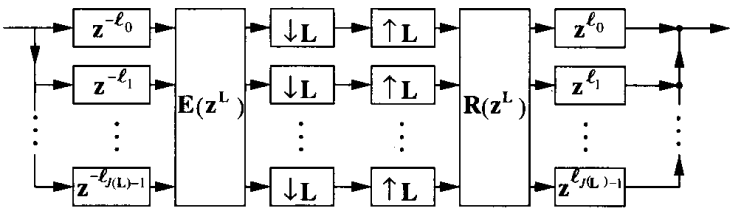

(b)

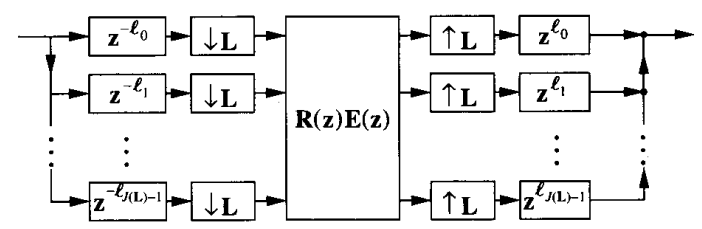

(c)

Fig. 4. MD uniform maximally decimated filter bank.

components. In the $\mathbf{z}$-domain, $X(\mathbf{z})$ is related to its polyphase components as

$$
\begin{array}{rlrl}
X(\mathbf{z}) & =\sum_{\mathbf{k} \in \mathcal{N}(\mathbf{L})} \mathbf{z}^{-\mathbf{k}} E_{\mathbf{k}}\left(\mathbf{z}^{\mathbf{L}}\right), \quad \text { (Type 1) } \\
\text { or } X(\mathbf{z}) & =\sum_{\mathbf{k} \in \mathcal{N}(\mathbf{L})} \mathbf{z}^{\mathbf{k}} R_{\mathbf{k}}\left(\mathbf{z}^{\mathbf{L}}\right), & \text { (Type 2) }
\end{array}
$$

Using the polyphase decomposition, we can represent the analysis filters and synthesis filters in the form

$$
H_{j}(\mathbf{z})=\sum_{k=0}^{J(\mathbf{L})-1} \mathbf{z}^{-\ell_{k}} E_{j, k}\left(\mathbf{z}^{\mathbf{L}}\right) \quad j=0, \cdots, J(\mathbf{L})-1
$$

and

$$
F_{j}(\mathbf{z})=\sum_{k=0}^{J(\mathbf{L})-1} \mathbf{z}^{\ell_{k}} R_{k, j}\left(\mathbf{z}^{\mathbf{L}}\right) \quad j=0, \cdots, J(\mathbf{L})-1 .
$$

Note that the vectors in $\mathcal{N}(\mathbf{L})$ are ordered as $\ell_{0}, \ell_{1}, \cdots$, $\ell_{J(L)-1}$ and $\ell_{0}$ is usually chosen to be the zero vector 0 . Then, we can redraw this system as in Fig. 4(b). The $J(\mathbf{L}) \times J(\mathbf{L})$ matrices $\mathbf{E}(\mathbf{z})$ and $\mathbf{R}(\mathbf{z})$ which have elements $E_{j, k}(\mathbf{z})$ and $R_{k, j}(\mathbf{z})$ are called the polyphase matrices of the analysis bank and the synthesis bank, respectively. MD Noble identities [25], [27], [28], as shown in Fig. 5, allow us to move the decimators and expanders across $\mathbf{E}\left(\mathbf{z}^{\mathbf{L}}\right)$ and $\mathbf{R}\left(\mathbf{z}^{\mathbf{L}}\right)$, respectively, and obtain Fig. 4(c). For this system to achieve PR, we must have $\mathbf{R}(\mathbf{z}) \mathbf{E}(\mathbf{z})=\mathbf{I}$. This implies that $\mathbf{R}(\mathbf{z})$ is invertible. For the case where all transfer functions are rational function in $\mathbf{z}$ with finite degree, $\mathbf{R}(\mathbf{z})$ being invertible implies that $\operatorname{det} \mathbf{R}(\mathbf{z})$ is not identically zero, but can be zero for some finite number of points in the $\mathbf{z}$-domain. We denote these points as $\mathbf{z}_{i}$. 


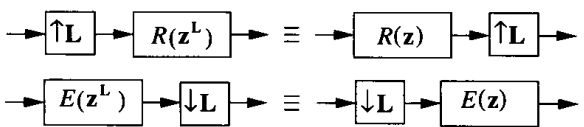

Fig. 5. MD Noble identities.

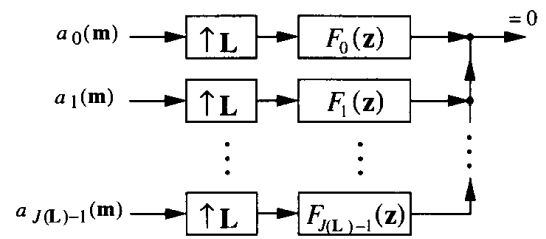

(a)

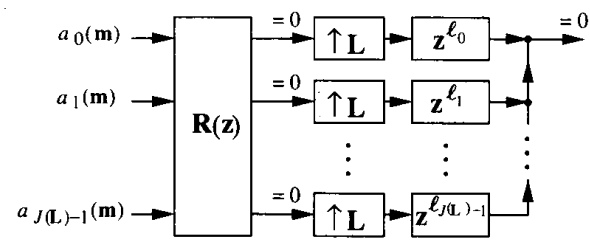

(b)

Fig. 6. MD uniform synthesis filter bank.

Now, suppose that $f_{k}(\mathbf{n}-\mathbf{L m})$ are not linearly independent. That is, there exist $a_{k}(\mathbf{m})$ which are not all zero such that

$$
\sum_{k=0}^{J(\mathbf{L})-1} \sum_{\mathbf{m} \in \mathcal{N}} a_{k}(\mathbf{m}) f_{k}(\mathbf{n}-\mathbf{L m})=0
$$

Let $a_{k}(\mathbf{m})$ be the inputs to the synthesis filter bank as shown in Fig. 6(a). Equation (5.5) implies that the output is identically zero. With Fig. 6(a) redrawn as in Fig. 6(b), it can be verified that the outputs of $\mathbf{R}(\mathbf{z})$ are also zero. Letting $A_{k}(\mathbf{z})$ denote the $\mathbf{z}$-transform of $a_{k}(\mathbf{m})$ and $\mathbf{a}(\mathbf{z})=$ $\left[A_{0}(\mathbf{z}) \cdots A_{J(\mathbf{L})-1}(\mathbf{z})\right]^{T}$, we have $\mathbf{R}(\mathbf{z}) \mathbf{a}(\mathbf{z})=\mathbf{0}$. Because $\operatorname{det} \mathbf{R}(\mathbf{z})$ is not identically zero, $\mathbf{a}(\mathbf{z})$ is zero almost everywhere except at $\mathbf{z}=\mathbf{z}_{i}$. For a finite number of points $\mathbf{z}_{i}$, $\mathbf{a}\left(\mathbf{z}_{i}\right)$ can be nonzero but finite. This implies $a_{k}(\mathbf{m})$ must be all zero. We hence conclude that $f_{k}(\mathbf{n}-\mathbf{L m})$ are linearly independent.

\section{B. Conversion to Uniform Filter Banks}

Next, we will show how to convert an MD nonuniform rational filter bank into a uniform filter bank with a larger number of channels while preserving the same set of $\eta_{k \mathbf{m}}(\mathbf{n})$. For the 1-D case, the problem of transforming a non-uniform filter bank into a uniform one has been addressed by Hoang and Vaidyanathan [4] and Kovačević and Vetterli [6]. This idea has been extended to the MD case and a graphical derivation has been given by Gopinath and Burrus [7]. The approach we present here will give the resulting uniform filter bank which is equivalent to that obtained in [7], and the corresponding subband signals as well. Furthermore, with our approach, we can show that the sets $\left\{\eta_{k \mathbf{m}}(\mathbf{n})\right\}$ and $\left\{\phi_{k \mathbf{m}}(\mathbf{n})\right\}$ of the converted uniform system are the same as those of the original nonuniform system.
Let us consider the set of functions $\eta_{k \mathbf{m}}(\mathbf{n})=f_{k}\left(\mathbf{P}_{k} \mathbf{n}-\right.$ $\left.\mathbf{Q}_{k} \mathbf{m}\right)$ of the MD nonuniform rational filter bank in Fig. 2. The division theorem [23, sec. 2.4.2] says that, with respect to a nonsingular integer matrix $\mathbf{M}$, every integer vector $\mathbf{n}$ can be uniquely expressed as $\mathbf{n}=\mathbf{M n}_{0}+\mathbf{k}$, for some $\mathbf{k} \in \mathcal{N}(\mathbf{M})$, and $\mathbf{n}_{0} \in \mathcal{N}$. Therefore, $\mathbf{Q}_{k} \mathbf{m}$ can be written as

$$
\mathbf{Q}_{k} \mathbf{m}=\mathbf{P}_{k} \mathbf{r}+\mathbf{p}, \quad \text { for } \mathbf{r} \in \mathcal{N} \text { and } \mathbf{p} \in \mathcal{N}\left(\mathbf{P}_{k}\right)
$$

where $\mathbf{r}$ and $\mathbf{p}$ are unique for a given $\mathbf{m}$. Let $f_{k, \mathbf{p}}(\mathbf{n})$ denote the $\mathbf{P}_{k}$-fold Type 2 polyphase components of $f_{k}(\mathbf{n})$, i.e., $f_{k, \mathbf{p}}(\mathbf{n}) \triangleq f_{k}\left(\mathbf{P}_{k} \mathbf{n}-\mathbf{p}\right)$, where $\mathbf{p} \in \mathcal{N}\left(\mathbf{P}_{k}\right)$. We then have

$f_{k}\left(\mathbf{P}_{k} \mathbf{n}-\mathbf{Q}_{k} \mathbf{m}\right)=f_{k}\left(\mathbf{P}_{k} \mathbf{n}-\mathbf{P}_{k} \mathbf{r}-\mathbf{p}\right)=f_{k, \mathbf{p}}(\mathbf{n}-\mathbf{r})$.

In other words, $f_{k}\left(\mathbf{P}_{k} \mathbf{n}-\mathbf{Q}_{k} \mathbf{m}\right)$ is the pth polyphase component of $f_{k}(\mathbf{n})$, delayed by $\mathbf{r}$.

Because $\mathbf{P}_{k}$ and $\mathbf{Q}_{k}$ are left coprime, there exist integer matrices $\mathbf{A}_{k}$ and $\mathbf{B}_{k}$ which are right coprime such that $\mathbf{P}_{k} \mathbf{A}_{k}+\mathbf{Q}_{k} \mathbf{B}_{k}=\mathbf{I}$ (generalized Bezout theorem [31]-[33]). Furthermore, there exist right coprime matrices $\mathbf{S}_{k}$ and $\mathbf{R}_{k}$ such that $\mathbf{P}_{k} \mathbf{S}_{k}=\mathbf{Q}_{k} \mathbf{R}_{k}$ and $J\left(\mathbf{S}_{k}\right)=J\left(\mathbf{Q}_{k}\right)$ [34]. For these $\mathbf{A}_{k}$ and $\mathbf{S}_{k}$, it is shown in Appendix $\mathbf{B}$ that any pair of $\mathbf{r}$ and $\mathbf{p}$ obtained in (5.6) satisfies

$$
\mathbf{r}=\mathbf{S}_{k} \mathbf{j}-\mathbf{A}_{k} \mathbf{p} \quad \text { for some } \mathbf{j} \in \mathcal{N}
$$

Now, (5.7) can be written as

$$
f_{k}\left(\mathbf{P}_{k} \mathbf{n}-\mathbf{Q}_{k} \mathbf{m}\right)=f_{k, \mathbf{p}}\left(\mathbf{n}+\mathbf{A}_{k} \mathbf{P}-\mathbf{S}_{k} \mathbf{j}\right) .
$$

Let $\mathbf{L}$ be a common right multiple (crm) [31] of $\mathbf{S}_{0}, \mathbf{S}_{1}, \cdots$ $\mathbf{S}_{M-1}$, and denote it as $\operatorname{crm}\left(\mathbf{S}_{0}, \cdots, \mathbf{S}_{M-1}\right)$. That is, $\mathbf{L}=$ $\mathbf{S}_{k} \mathbf{T}_{k}$ for all $k$, where $\mathbf{T}_{k}$ are integer matrices. In particular, we can choose a least common right multiple (lcrm) [31]. Next, we use the division theorem again to write $\mathbf{j}$ as

$$
\mathbf{j}=\mathbf{T}_{k} \mathbf{i}+\mathbf{t} \quad \text { for } \mathbf{i} \in \mathcal{N} \text { and } \mathbf{t} \in \mathcal{N}\left(\mathbf{T}_{k}\right) .
$$

Note that $\mathbf{i}$ and $\mathbf{t}$ are unique for a given $\mathbf{j}$. Substituting this into (5.9), we get

$f_{k}\left(\mathbf{P}_{k} \mathbf{n}-\mathbf{Q}_{k} \mathbf{m}\right)=f_{k, \mathbf{p}}\left(\mathbf{n}+\mathbf{A}_{k} \mathbf{p}-\mathbf{S}_{k} \mathbf{t}-\mathbf{L} \mathbf{i}\right)=f_{k \mathbf{p t}}^{\prime}(\mathbf{n}-\mathbf{L i})$

where $f_{k \mathbf{p t}}^{\prime}(\mathbf{n})$ is defined as $f_{k \mathbf{p t}}^{\prime}(\mathbf{n}) \triangleq f_{k, \mathbf{p}}\left(\mathbf{n}+\mathbf{A}_{k} \mathbf{p}-\mathbf{S}_{k} \mathbf{t}\right)$.

Combining (5.6), (5.8) and (5.10), we can get the following expression for $\mathbf{m}$

$$
\mathbf{m}=\mathbf{R}_{k} \mathbf{T}_{k} \mathbf{i}+\mathbf{R}_{k} \mathbf{t}+\mathbf{B}_{k} \mathbf{p} .
$$

It can be shown that (5.6), (5.8) and (5.10) altogether define a one-to-one and onto mapping from a single index $\mathbf{m} \in \mathcal{N}$ to a triple index $(\mathbf{p}, \mathbf{t}, \mathbf{i})$ where $\mathbf{p} \in \mathcal{N}\left(\mathbf{P}_{k}\right), \mathbf{t} \in \mathcal{N}\left(\mathbf{T}_{k}\right)$ and $\mathbf{i} \in \mathcal{N}$. Using the same mapping to map subband signals $x_{k}(\mathbf{m})$ to $x_{k \mathbf{p t}}(\mathbf{i})$, we can rewrite $(3.10)$ as

$$
\widehat{x}(\mathbf{n})=\sum_{k=0}^{M-1} \sum_{\mathbf{p} \in \mathcal{N}\left(\mathbf{P}_{k}\right)} \sum_{\mathbf{t} \in \mathcal{N}\left(\mathbf{T}_{k}\right)} \sum_{\mathbf{i} \in \mathcal{N}} x_{k \mathbf{p}}(\mathbf{i}) f_{k \mathbf{p t}}^{\prime}(\mathbf{n}-\mathbf{L i}) .
$$

Note that the summation over $\mathbf{m}$ is replaced by a triple summation over $\mathbf{p}, t$, and $\mathbf{i}$. 


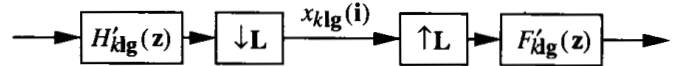

Fig. 7. One branch of an MD uniformly decimated filter bank converted from a nonuniform filter bank.

We can preform similar operations on the analysis filter bank also. We use $h_{k, \mathbf{p}}(\mathbf{n})$ to denote the $\mathbf{P}_{k}$-fold Type 1 polyphase components of $h_{k}(\mathbf{n})$, i.e., $h_{k, \mathbf{p}}(\mathbf{n}) \triangleq h_{k}\left(\mathbf{P}_{k} \mathbf{n}+\mathbf{p}\right)$, and rewrite $h_{k}\left(\mathbf{Q}_{k} \mathbf{m}-\mathbf{P}_{k} \mathbf{n}\right)$ as follows

$$
\begin{aligned}
h_{k}\left(\mathbf{Q}_{k} \mathbf{m}-\mathbf{P}_{k} \mathbf{n}\right) & =h_{k}\left(\mathbf{P}_{k} \mathbf{r}+\mathbf{p}-\mathbf{P}_{k} \mathbf{n}\right) \\
& =h_{k, \mathbf{p}}(\mathbf{r}-\mathbf{n}) \\
& =h_{k, \mathbf{p}}\left(\mathbf{S}_{k} \mathbf{j}-\mathbf{A}_{k} \mathbf{p}-\mathbf{n}\right) \\
& =h_{k, \mathbf{p}}\left(\mathbf{L i}+\mathbf{S}_{k} \mathbf{t}-\mathbf{A}_{k} \mathbf{p}-\mathbf{n}\right) \\
& =h_{k \mathbf{p t}}^{\prime}(\mathbf{L} \mathbf{i}-\mathbf{n})
\end{aligned}
$$

where $h_{k \mathbf{p t}}^{\prime}(\mathbf{n})$ is defined as $h_{k \mathbf{p t}}^{\prime}(\mathbf{n}) \triangleq h_{k, \mathbf{p}}\left(\mathbf{n}-\mathbf{A}_{k} \mathbf{p}+\mathbf{S}_{k} \mathbf{t}\right)$. So, (3.8) becomes

$$
\begin{aligned}
x_{k \mathbf{p t}}(\mathbf{i}) & =\sum_{\mathbf{n} \in \mathcal{N}} x(\mathbf{n}) h_{k \mathbf{p t}}^{\prime}(\mathbf{L i}-\mathbf{n}) \\
k & =0, \cdots, M-1, \mathbf{p} \in \mathcal{N}\left(\mathbf{P}_{k}\right), \mathbf{t} \in \mathcal{N}\left(\mathbf{T}_{k}\right) .
\end{aligned}
$$

Looking at (5.13) and (5.15), we realize that the original nonuniformly decimated analysis/synthesis filter bank has been converted into a uniformly decimated filter bank with decimation ratio $\mathbf{L}$. The channels are now labeled by three indices $k$, $\mathbf{p}$ and $\mathbf{t}$. One channel (the ' $k$ ptth' channel) of this uniform filter bank is shown in Fig. 7. The new subband signals are $x_{k \mathbf{p t}}(\mathbf{i})$, where $\mathbf{i}$ is the space domain index. Because $x_{k \mathbf{p t}}(\mathbf{i})=$ $x_{k}(\mathbf{m})$ where $\mathbf{m}$ is obtainable as in (5.12), each $x_{k \mathbf{p t}}(\mathbf{i})$ is a $\mathbf{R}_{k} \mathbf{T}_{k}$-fold polyphase component (with proper shifts) of the original subband signal $x_{k}(\mathbf{m})$. Similarly, the new analysis filters $H_{k \mathbf{p t}}^{\prime}(\mathbf{z})$ and the synthesis filters $F_{k \mathbf{p t}}^{\prime}(\mathbf{z})$ are the $\mathbf{P}_{k^{-}}$ fold polyphase components (with proper shifts) of $H_{k}(\mathbf{z})$ and $F_{k}(\mathbf{z})$, respectively. The corresponding new $\phi_{k p t i}^{\prime}(\mathbf{n})$ and $\eta_{k \mathbf{p t i}}^{\prime}(\mathbf{n})$ are $h_{k \mathrm{pt}}^{\prime}(\mathbf{L i}-\mathbf{n})$ and $f_{k \mathrm{pt}}^{\prime}(\mathbf{n}-\mathbf{L i})$, respectively From (5.11) and (5.14), we see that these are indeed the $\phi_{k \mathbf{m}}(\mathbf{n})$ and $\eta_{k \mathbf{m}}(\mathbf{n})$ of the original rational filter bank. In other words, $\left\{\phi_{k \mathbf{m}}(\mathbf{n})\right\}=\left\{\phi_{k \mathbf{p t i}}^{\prime}(\mathbf{n})\right\}$ and $\left\{\eta_{k \mathbf{m}}(\mathbf{n})\right\}=$ $\left\{\eta_{k \mathbf{p t i}}^{\prime}(\mathbf{n})\right\}$. Counting the number of channels in this extended uniform filter bank, we get $\sum_{k=0}^{M-1} J\left(\mathbf{P}_{k}\right) J\left(\mathbf{T}_{k}\right)=$ $\sum_{k=0}^{M-1} J\left(\mathbf{P}_{k}\right) J(\mathbf{L}) / J\left(\mathbf{S}_{k}\right)=J(\mathbf{L}) \sum_{k=0}^{M=1} J\left(\mathbf{P}_{k}\right) / J\left(\mathbf{Q}_{k}\right)=$ $J(\mathbf{L})$, so the resulting uniform filter bank is also maximally decimated. It is also clear that the resulting uniform system achieves PR if and only if the original nonuniform system achieves PR. For the uniform case, we have already shown that $\eta_{k \mathbf{p t i}}^{\prime}(\mathbf{n})$ are linearly independent if $\mathrm{PR}$ is possible. Because $\left\{\eta_{k \mathbf{m}}(\mathbf{n})\right\}$ and $\left\{\eta_{k \mathbf{p t i}}^{\prime}(\mathbf{n})\right\}$ are identical, $\eta_{k \mathbf{m}}(\mathbf{n})$ of the nonuniform rational filter bank should also be linearly independent.

Remark: This approach of converting nonuniform filter banks places in evidence the resulting uniform filter bank, the corresponding new $\phi_{k \mathbf{p t i}}^{\prime}(\mathbf{n})$ and $\eta_{k \mathrm{pti}}^{\prime}(\mathbf{n})$, and the expressions for the corresponding subband signals as well. The expressions of resulting subband signals will be useful in the next section for the derivation of MD subband convolution.

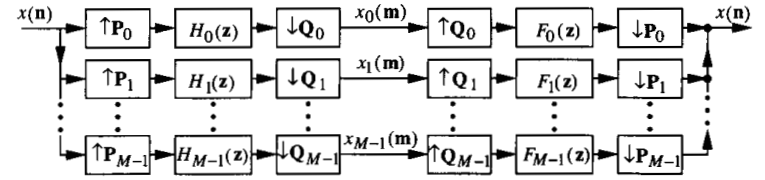

(a)

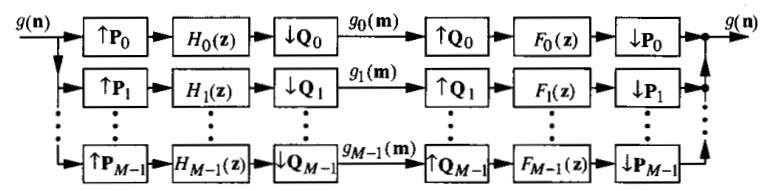

(b)

Fig. 8. MD PR rational filter bank. (a) With input $x(\mathbf{n})$. (b) With input $g(\mathbf{n})$.

\section{MD SubBand CONvolution TheOREM}

For the 1-D case, it has been shown that the convolution of two signals can be obtained by convolving corresponding subband signals and adding these up [20]. This result, which is called the subband convolution theorem, holds for both orthonormal filter banks and biorthonormal filter banks. When signals are to be quantized, subband convolution provides better performance (less quantization error) than direct convolution. We will show that the same result can be obtained by starting from the generalized Parseval's relation in Section II. We can therefore extend the subband convolution theorem to the most general filter banks, namely, MD nonuniform filter banks with rational decimation matrices.

Suppose we want to compute the convolution of two MD signals $x(\mathbf{n})$ and $g^{*}(-\mathbf{n})$

$$
w(\mathbf{k})=x(\mathbf{k}) * g^{*}(-\mathbf{k}) \triangleq \sum_{\mathbf{n} \in \mathcal{N}} x(\mathbf{n}) g^{*}(\mathbf{n}-\mathbf{k}) .
$$

This is also called the deterministic cross correlation between $x(\mathbf{n})$ and $g(\mathbf{n})$. Suppose we feed $x(\mathbf{n})$ and $g(\mathbf{n})$ into a PR filter bank where the synthesis filters are orthonormal, i.e., (3.15) is satisfied. This is shown in Fig. 8(a) and Fig. 8(b), where $x_{k}(\mathbf{m})$ and $g_{k}(\mathbf{m})$ denote the corresponding subband signals. Using the same correspondence between vector space and filter banks as mentioned in Section III, we can apply Corollary 4.1 to obtain

$$
w(\mathbf{0})=\sum_{\mathbf{n} \in \mathcal{N}} x(\mathbf{n}) g^{*}(\mathbf{n})=\sum_{k=0}^{M-1} \sum_{\mathbf{m} \in \mathcal{N}} x_{k}(\mathbf{m}) g_{k}^{*}(\mathbf{m}) .
$$

In other words, the inner product of $x(\mathbf{n})$ and $g(\mathbf{n})$ can be obtained by computing the inner product of each pair of $x_{k}(\mathbf{m})$ and $g_{k}(\mathbf{m})$, and adding the results.

Energy conservation and orthonormality: If we let $g(\mathbf{n})=$ $x(\mathbf{n})$ in (6.2), we get the energy conservation equation $\sum_{\mathbf{n} \in \mathcal{N}}|x(\mathbf{n})|^{2}=\sum_{k=0}^{M-1} \sum_{\mathbf{m} \in \mathcal{N}}\left|x_{k}(\mathbf{m})\right|^{2}$. Furthermore, using Corollary 4.2, we can show that the energy is preserved in the subband signals if and only if the PR filter bank is an orthonormal filter bank.

We proceed to relate the inner product in (6.2) to convolution. Equation (6.2) gives only one sample of the convolution, i.e., $w(\mathbf{0})$. We can obtain $w(\mathbf{k})$ for other values of $\mathbf{k}$ by shifting the input in Fig. 8(b) by k. Let us consider the case of uniform 


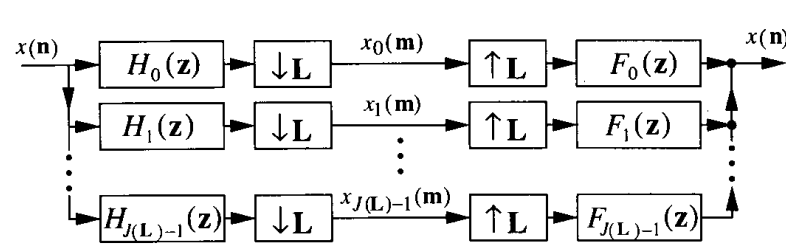

(a)

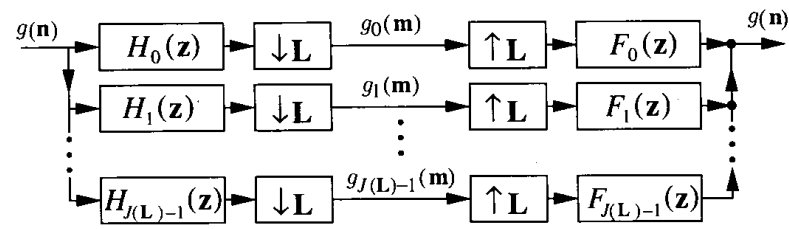

(b)

Fig. 9. MD PR uniform filter bank. (a) With input $x(\mathbf{n})$. (b) With input $g(\mathbf{n})$.

filter banks first. For this case, all $\mathbf{P}_{k}$ are identity matrices and all $\mathbf{Q}_{k}$ are equal to $\mathbf{L}$, as shown in Fig. 9(a) and Fig. 9(b). Due to maximal decimation, the decimation ratio $J(\mathbf{L})$ is equal to the number of channels. Let us conceptually shift $g(\mathbf{n})$ in Fig. 9 (b) by Lk. That is, we let $g^{\prime}(\mathbf{n})=g(\mathbf{n}-\mathbf{L k})$ and let $g_{k}^{\prime}(\mathbf{m})$ denote the corresponding subband signals. It can be verified that $g_{k}^{\prime}(\mathbf{m})=g_{k}(\mathbf{m}-\mathbf{k})$. So

$$
\begin{aligned}
w(\mathbf{L k}) & =\sum_{\mathbf{n} \in \mathcal{N}} x(\mathbf{n}) g^{*}(\mathbf{n}-\mathbf{L k})=\sum_{\mathbf{n} \in \mathcal{N}} x(\mathbf{n}) g^{\prime *}(\mathbf{n}) \\
& =\sum_{k=0}^{J(\mathbf{L})-1} \sum_{\mathbf{m} \in \mathcal{N}} x_{k}(\mathbf{m}) g_{k}^{* *}(\mathbf{m}) \\
& =\sum_{k=0}^{J(\mathbf{L})-1} \sum_{\mathbf{m} \in \mathcal{N}} x_{k}(\mathbf{m}) g_{k}^{*}(\mathbf{m}-\mathbf{k}) .
\end{aligned}
$$

This can be written as

$$
[\underbrace{x(\mathbf{k}) * g^{*}(-\mathbf{k})}_{w(\mathbf{k})}]_{\downarrow \mathbf{L}}=\sum_{k=0}^{J(\mathbf{L})-1}\left[x_{k}(\mathbf{k}) * g_{k}^{*}(-\mathbf{k})\right]
$$

where $[\cdot]_{\downarrow L}$ denotes the result of $\mathbf{L}$-fold decimation. In other words, the $\mathbf{L}$-fold decimated version of $w(\mathbf{k})$ is obtainable by adding up the convolution of corresponding subband signals. This is the MD subband convolution theorem for the case of uniform filter banks.

Note that (6.4) only gives the $\mathbf{L}$-fold decimated version of $w(\mathbf{k})$, i.e., the 0 th polyphase components of $w(\mathbf{k})$. To obtain other polyphase components of $w(\mathbf{k})$, we can shift the input in Fig. 9(b) by $\mathbf{j}$, where $\mathbf{j} \in \mathcal{N}(\mathbf{L})$, and repeat the computation in (6.4) again. More specifically, letting $g^{(\mathbf{j})}(\mathbf{n})=g(\mathbf{n}-\mathbf{j})$ and $g_{k}^{(\mathbf{j})}(\mathbf{m})$ denote the corresponding subband signals, we can obtain the jth polyphase components of $w(\mathbf{k})$ by

$$
\begin{aligned}
w(\mathbf{L} \mathbf{k}+\mathbf{j}) & =\sum_{\mathbf{n} \in \mathcal{N}} x(\mathbf{n}) g^{*}(\mathbf{n}-\mathbf{L k}-\mathbf{j}) \\
& =\sum_{\mathbf{n} \in \mathcal{N}} x(\mathbf{n})\left[g^{(\mathbf{j})}(\mathbf{n}-\mathbf{L} \mathbf{k})\right]^{*} \\
& =\sum_{k=0}^{J(\mathbf{L})-1} \sum_{\mathbf{m} \in \mathcal{N}} x_{k}(\mathbf{m})\left[g_{k}^{(\mathbf{j})}(\mathbf{m}-\mathbf{k})\right]^{*}
\end{aligned}
$$

or $w(\mathbf{L k}+\mathbf{j})=\left[x(\mathbf{k}) *\left[g^{(\mathbf{j})}(-\mathbf{k})\right]^{*}\right]_{\downarrow} \mathbf{L}=\sum_{k=0}^{M-1} x_{k}(\mathbf{k}) *$ $\left[g_{k}^{(\mathbf{j})}(-\mathbf{k})\right]^{*}$. The complete convolution $w(\mathbf{k})$ is obtainable by recombining these $w(\mathbf{L} \mathbf{k}+\mathbf{j})$, where $\mathbf{j} \in \mathcal{N}(\mathbf{L})$.

We can now extend the MD subband convolution theorem to the nonuniform case. Reconsider the MD nonuniform filter bank in Fig. 8. It has been shown in Section V that this nonuniform filter bank can be converted to an equivalent uniform filter bank. Using the notations in Section V, we modify (6.3) to get the following equation for resulting uniform filter bank

$$
w(\mathbf{L k})=\sum_{k=0}^{M-1} \sum_{\mathbf{p} \in \mathcal{N}\left(\mathbf{P}_{k}\right)} \sum_{\mathbf{t} \in \mathcal{N}\left(\mathbf{T}_{k}\right)} \sum_{\mathbf{i} \in \mathcal{N}} x_{k \mathbf{p t}}(\mathbf{i}) g_{k \mathbf{p} \mathbf{t}}^{*}(\mathbf{i}-\mathbf{k}) .
$$

Rewriting the triple summation over $\mathbf{p}, \mathbf{t}$, and $\mathbf{i}$ into one single summation over $\mathbf{m}$ using (5.12), we get

$$
w(\mathbf{L k})=\sum_{k=0}^{M-1} \sum_{\mathbf{m} \in \mathcal{N}} x_{k}(\mathbf{m}) g_{k}^{*}\left(\mathbf{m}-\mathbf{R}_{k} \mathbf{T}_{k} \mathbf{k}\right)
$$

which is the same as $\left[x(\mathbf{k}) * g^{*}(-\mathbf{k})\right]_{\downarrow \mathbf{L}}=\sum_{k=0}^{M-1}\left[x_{k}(\mathbf{k}) *\right.$ $\left.g_{k}^{*}(-\mathbf{k})\right]_{\downarrow \mathbf{R}_{k}} \mathbf{T}_{k}$. Again, to obtain the complete $w(\mathbf{k})$, we can shift $g(\mathbf{n})$ by $\mathbf{j}$, where $\mathbf{j} \in \mathcal{N}(\mathbf{L})$, to compute the jth polyphase component and then recombine these results. Summarizing, we have derived the following theorem using vector space notations:

Theorem 8: Subband convolution theorem (orthonormal case): Suppose we have an MD. nonuniform rational filter bank which achieves PR and the synthesis filters are orthonormal. The $\mathbf{L}$-fold decimated version of the convolution of $x(\mathbf{k})$ and $g^{*}(-\mathbf{k})$ can be obtained by convolving corresponding subband signals, decimating each convolution by $\mathbf{R}_{k} \mathbf{T}_{k}$, and adding up the results. In other words

$$
\left[x(\mathbf{k}) * g^{*}(-\mathbf{k})\right]_{\downarrow \mathbf{L}}=\sum_{k=0}^{M-1}\left[x_{k}(\mathbf{k}) * g_{k}^{*}(-\mathbf{k})\right]_{\downarrow \mathbf{R}_{k} \mathbf{T}_{k}}
$$

\section{A. Generalization to the Biorthonormal Case}

The MD subband convolution theorem can also be extended to the case of biorthonormal filter banks. Starting from Theorem 4, the generalized Parseval's relation for the biorthonormal case, we can derive the following:

Theorem 9: Subband convolution theorem (biorthonormalcase): Suppose we have an MD nonuniform rational filter bank which achieves PR. In other words, the analysis and synthesis filters are biorthonormal. We feed $x(\mathbf{n})$ into to this filter bank and let $x_{k}(\mathbf{m})$ denote the resulting subband signals. Interchanging the analysis and the synthesis filters, we get another PR filter bank (Corollary 6.1). We feed $y(\mathbf{n})$ into to this new filter bank and let $y_{k}(\mathbf{m})$ denote the resulting subband signals, as shown in Fig. 10. Let $w(\mathbf{k})$ denote the convolution of $x(\mathbf{k})$ and $y(\mathbf{k})$. The $\mathbf{L}$-fold decimated version of $w(\mathbf{k})$ can be obtained by convolving corresponding subband signals, decimating each convolution by $\mathbf{R}_{k} \mathbf{T}_{k}$, and adding 


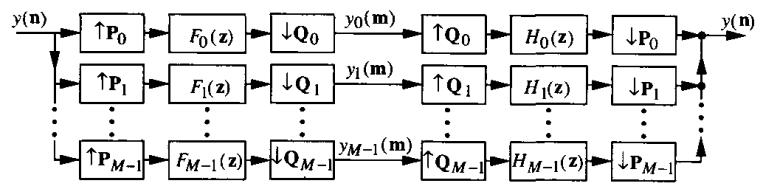

Fig. 10. MD PR rational filter bank, with interchanged analysis and synthesis filters, and with input $y(\mathbf{n})$.

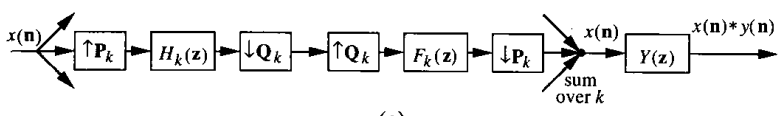

(a)

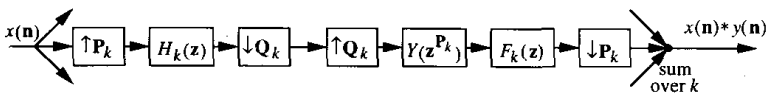

(b)

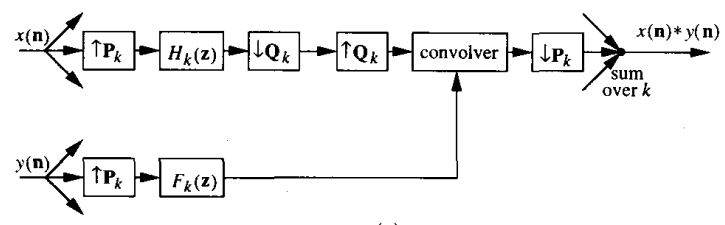

(c)

Fig. 11. MD subband convolution of $x(\mathbf{n})$ and $y(\mathbf{n})$.

up the results. In other words

$$
[\underbrace{x(\mathbf{k}) * y(\mathbf{k})}_{w(\mathbf{k})}]_{\downarrow \mathbf{L}}=\sum_{k=0}^{M-1}\left[x_{k}(\mathbf{k}) * y_{k}(\mathbf{k})\right]_{\downarrow \mathbf{R}_{k}} \mathbf{T}_{k} .
$$

\section{Remarks:}

1) The other polyphase components of $w(\mathbf{k})$ can be obtained by advancing $y(\mathbf{n})$ by $\mathbf{j}$, say $y^{(\mathbf{j})}(\mathbf{n})=y(\mathbf{n}+$ $\mathrm{j}$ ), and repeating (6.9) for the corresponding subband signals $y_{k}^{(\mathbf{j})}(\mathbf{m})$.

2) Note that the convolution, rather than the deterministic cross-correlation, of $x(\mathbf{n})$ and $y(\mathbf{n})$ is obtained in this theorem.

3) For the case that the synthesis filters are indeed orthonormal, we know $f_{k}(\mathbf{n})=h_{k}^{*}(-\mathbf{n})$ (Theorem 5). Therefore, according to the above theorem, $y(\mathbf{n})$ should be analyzed using filters $h_{k}^{*}(-\mathbf{n})$. Letting $g(\mathbf{n})=y^{*}(-\mathbf{n})$, we see this is same as analyzing $g(\mathbf{n})$ using filters $h_{k}(\mathbf{n})$. Now, $x(\mathbf{k}) * g^{*}(-\mathbf{k})=x(\mathbf{k}) * y(\mathbf{k})$, and Theorem 8 is hence obtained as a special case of Theorem 9 .

The proof of Theorem 9 is similar to the orthonormal case, so it is omitted here. Instead, we will give a graphical proof as follows. This will also give an implementation of the subband convolution. First, let us treat $y(\mathbf{n})$ as an MD filter. As shown in Fig. 11(a), the convolution of $x(\mathbf{n})$ and $y(\mathbf{n})$ can be obtained by passing $x(\mathbf{n})$ through a PR filter bank and an MD filter $Y(\mathbf{z})$, where $Y(\mathbf{z})$ is the z-transform of $y(\mathbf{n})$. For simplicity, only one channel in each filter bank is shown is shown in this and the following figures. Using Noble identity, we can move $Y(\mathbf{z})$ to the left of $\mathbf{P}_{k}$-fold decimators to obtain Fig. 11(b). With $y(\mathbf{n})$ treated as an MD

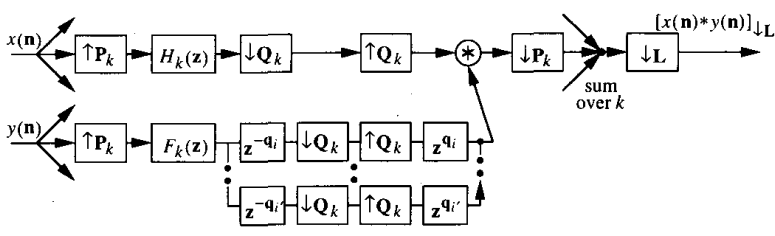

(a)

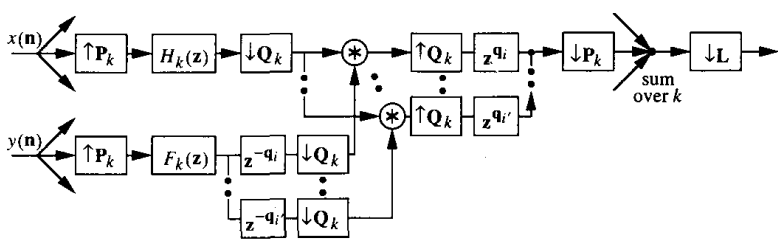

(b)

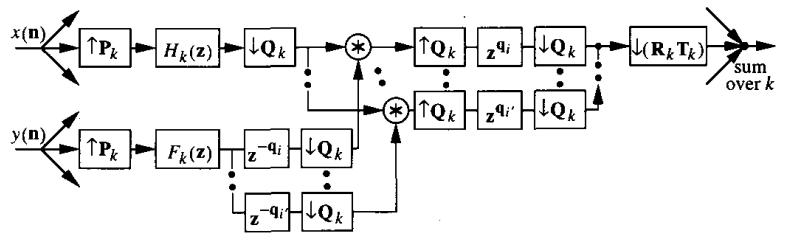

(c)

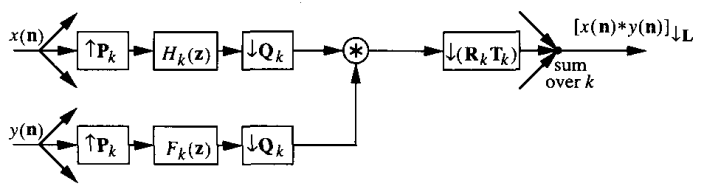

(d)

Fig. 12. Obtaining the L-fold decimated version of $x(\mathbf{n}) * y(\mathbf{n})$.

signal again, Fig. 11(b) can be redrawn as Fig. 11(c), which gives an implementation of MD subband convolution.

To prove the subband convolution theorem, we cascade the system in Fig. 11 by an $\mathbf{L}$-fold decimator to obtain $[x(\mathbf{n}) * y(\mathbf{n})]_{\downarrow \mathbf{L}}$. Inserting a delay-chain system [34] after each filter $F_{k}(\mathbf{z})$, we obtain Fig. 12(a), where the notation $\circledast$ stands for a convolver. The delay-chain system has $J\left(\mathbf{q}_{k}\right)$ branches and $\mathbf{q}_{i}$ are the elements in $\mathcal{N}\left(\mathbf{Q}_{k}\right)$. It can be verified that a delay-chain system is a perfect reconstruction system so that Fig. 12(a) is indeed equivalent to Fig. 11(c) followed by an Lfold decimator. Moving convolvers to the left, we can redraw Fig. 12(a) to get Fig. 12(b). Moving the $\mathbf{L}$-fold decimator to the left and combining it with the $\mathbf{P}_{k}$-fold decimator, we obtain Fig. 12(c), because $\mathbf{P}_{k} \mathbf{L}=\mathbf{P}_{k} \mathbf{S}_{k} \mathbf{T}_{k}=\mathbf{Q}_{k} \mathbf{R}_{k} \mathbf{T}_{k}$. It can be shown that a filter $h(\mathbf{n})$ preceded by a $\mathbf{Q}_{k}$-fold expander and followed by a $\mathbf{Q}_{k}$-fold decimator is equivalent to a filter with impulse response $h\left(\mathbf{Q}_{k} \mathbf{n}\right)$. Therefore, each $\mathbf{z}^{\mathbf{q}_{i}}$ preceded by a $\mathbf{Q}_{k}$-fold expander and followed by a $\mathbf{Q}_{k}$-fold decimator has transfer function zero unless $\mathbf{q}_{i}=\mathbf{0}$. Hence, we can remove $J\left(\mathbf{Q}_{k}\right)-1$ branches in each channel and obtain Fig. 12(d). This indeed gives the result stated in Theorem 9.

\section{B. Coding Gain of Subband Convolution (The General Biorthonormal Case)}

The main advantage of subband convolution is that we can quantize the subband signals according to the signal variance 
in each subband and other perceptual considerations, as in the traditional subband coding [35]. For a fixed bit rate, the result of subband convolution is more accurate than that of direct convolution, and we hence obtain a coding gain over direct convolution. To analyze the coding gain for MD subband convolution, we shall make the following assumptions:

1) We assume $x(\mathbf{n})$ is an MD zero-mean wide-sensestationary (WSS) random process and $y(\mathbf{n})$ is an MD deterministic signal.

2) Given that $x(\mathbf{n})$ is WSS, $x_{k}(\mathbf{m})$ is in general not WSS, but cyclo-wide-sense-stationary with period $\mathbf{Q}_{k}^{-1} \operatorname{lcrm}\left(\mathbf{P}_{k}, \mathbf{Q}_{k}\right)$ [34]. However, we shall assume that $x_{k}(\mathbf{m})$ are all zero-mean WSS with variance $\sigma_{x_{k}}^{2}$. It can be shown that this assumption is true if $\mathbf{P}_{k}$ are unimodular (i.e., with determinant \pm 1 ) or all the analysis filters $H_{k}(\mathbf{z})$ are ideal filters. The reason is similar to that of the 1-D case [36]. For the case that filters are not ideal but having large stopband attenuation, $x_{k}(\mathbf{m})$ are approximately WSS.

3) Let $q_{k}(\mathbf{m})$ denote the quantization error for $x_{k}(\mathbf{m})$. That is, $x_{k}(\mathbf{m})$ is quantized to $x_{k}(\mathbf{m})+q_{k}(\mathbf{m})$. Assume $q_{k}(\mathbf{m})$ are zero-mean WSS with variance $\sigma_{q_{k}}^{2}$. Assume that all $q_{k}(\mathbf{m})$ are white and uncorrelated, and that all $q_{k}(\mathbf{m})$ are uncorrelated to $x_{k}(\mathbf{m})$. With $b_{k}$ denoting the number of bits assigned to the quantizer for $x_{k}(\mathbf{m}), \sigma_{q_{k}}^{2}$ is related to $\sigma_{x_{k}}^{2}$ as

$$
\sigma_{q_{k}}^{2}=c \sigma_{x_{k}}^{2} 2^{-2 b_{k}}
$$

for some constant $c$ [22, appendix C], [35].

Under these assumptions, we can compute the error in $w(\mathbf{L k})$ due to quantization

$$
q(\mathbf{k})=\sum_{k=0}^{M-1}\left[q_{k}(\mathbf{k}) * y_{k}(\mathbf{k})\right]_{\downarrow \mathbf{R}_{k} \mathbf{T}_{k}}
$$

We obtain that the variance of $q_{k}(\mathbf{k}) * y_{k}(\mathbf{k})$ is $\sigma_{q_{k}}^{2} \sum_{\mathbf{i} \in \mathcal{N}}\left|y_{k}(\mathbf{i})\right|^{2}$ and the variance of $q(\mathbf{k})$ is

$\sigma_{q(\mathbf{k})}^{2}=\sum_{k=0}^{M-1} \sigma_{q_{k}}^{2} \sum_{\mathbf{i} \in \mathcal{N}}\left|y_{k}(\mathbf{i})\right|^{2}=c \sum_{k=0}^{M-1} 2^{-2 b_{k}} \sigma_{x_{k}}^{2} \sum_{\mathbf{i} \in \mathcal{N}}\left|y_{k}(\mathbf{i})\right|^{2}$.

Note that the $\mathbf{R}_{k} \mathbf{T}_{k}$-fold decimation does not change the variance. To compute the whole $w(\mathbf{k})$, we need to advance $y(\mathbf{n})$ by $\mathbf{j}$ and repeat $(6.9)$ for $J(\mathbf{L})$ times. So, we need to average (6.12) over all $\mathbf{j} \in \mathcal{N}(\mathbf{L})$ to obtain the variance of overall quantization error

$$
\sigma_{q}^{2}=\frac{c}{J(\mathbf{L})} \sum_{k=0}^{M-1} 2^{-2 b_{k}} \sigma_{x_{k}}^{2} \sum_{\mathbf{j} \in \mathcal{N}(\mathbf{L})} \sum_{\mathbf{i} \in \mathcal{N}}\left|y_{k}^{(\mathbf{j})}(\mathbf{i})\right|^{2} .
$$

Define

$$
\alpha_{k}^{2} \triangleq \frac{M}{J(\mathbf{L})} \sum_{\mathbf{j} \in \mathcal{N}(\mathbf{L})} \sum_{\mathbf{i} \in \mathcal{N}}\left|y_{k}^{(\mathbf{j})}(\mathbf{i})\right|^{2}
$$

which can be shown to be proportional to the energy of $y_{k}(\mathbf{m})$ We can rewrite $(6.13)$ as

$$
\sigma_{q}^{2}=\frac{c}{M} \sum_{k=0}^{M-1} 2^{-2 b_{k}} \sigma_{x_{k}}^{2} \alpha_{k}^{2}
$$

Let $b$ denote the overall bit rate, so $b=$ $\sum_{k=0}^{M-1} b_{k} J\left(\mathbf{P}_{k}\right) / J\left(\mathbf{Q}_{k}\right)$. Under the constraint of fixed $b$, it can be shown that $\sigma_{q}^{2}$ is minimized when

$$
\begin{aligned}
b_{k}= & b+0.5 \log _{2}\left(\frac{J\left(\mathbf{Q}_{k}\right)}{J\left(\mathbf{P}_{k}\right)} \sigma_{x_{k}}^{2} \alpha_{k}^{2}\right) \\
& -0.5 \sum_{i=0}^{M-1} \frac{\log _{2}\left(\frac{J\left(\mathbf{Q}_{i}\right)}{J\left(\mathbf{P}_{i}\right)} \sigma_{x_{i}}^{2} \alpha_{i}^{2}\right)}{J\left(\mathbf{Q}_{i}\right) / J\left(\mathbf{P}_{i}\right)} .
\end{aligned}
$$

The proof of this uses the Lagrange multiplier method and is similar to that in [20]. Substituting (6.16) into (6.10), we can see that $\sigma_{q}^{2}$ is minimized when $\sigma_{q_{k}}^{2}$ is proportional to $J\left(\mathbf{P}_{k}\right) / \alpha_{k}^{2} J\left(\mathbf{Q}_{k}\right)$. The minimized error variance under the above optimal bit allocation is

$$
\sigma_{q, \mathrm{opt}}^{2}=\frac{c 2^{-2 b}}{M} \prod_{k=0}^{M-1}\left(\frac{J\left(\mathbf{Q}_{k}\right)}{J\left(\mathbf{P}_{k}\right)} \sigma_{\boldsymbol{x}_{k}}^{2} \alpha_{k}^{2}\right)^{J\left(\mathbf{P}_{k}\right) / J\left(\mathbf{Q}_{k}\right)} .
$$

Comparing this with direct convolution, where the error variance is $\sigma_{q \text {,direct }}^{2}=c 2^{-2 b} \sigma_{x}^{2} \sum_{\mathbf{i} \in \mathcal{N}}|y(\mathbf{i})|^{2}$, we see that the coding gain of subband convolution over direct convolution is

$$
G_{\mathrm{opt}}=\frac{\sigma_{q, \mathrm{direct}}^{2}}{\sigma_{q, \mathrm{opt}}^{2}}=\frac{\sigma_{x}^{2} \sum_{\mathbf{i} \in \mathcal{N}}|y(\mathbf{i})|^{2}}{\frac{1}{M} \prod_{k=0}^{M-1}\left(\frac{J\left(\mathbf{Q}_{k}\right)}{J\left(\mathbf{P}_{k}\right)} \sigma_{x_{k}}^{2} \alpha_{k}^{2}\right)^{J\left(\mathbf{P}_{k}\right) / J\left(\mathbf{Q}_{k}\right)}} .
$$

\section{Remarks:}

1) For the orthonormal case, it can be shown that the coding obtained above is always greater or equal to unity. It is unity if and only if $\sigma_{x_{k}}^{2}$ and $\alpha_{k}^{2} J\left(\mathbf{Q}_{k}\right) / J\left(\mathbf{P}_{k}\right)$ are both independent of $k$.

2) For the orthonormal case, if we let $y(\mathbf{n})=\delta(\mathbf{n})$, the above analysis can be used to get the optimal coding gain for MD subband coding, i.e., the coding gain of quantizing subband signals $x_{k}(\mathbf{m})$ over directly quantizing $x(\mathbf{n})$. When $y(\mathbf{n})=\delta(\mathbf{n})$, it can be shown that $\alpha_{k}^{2}=M J\left(\mathbf{P}_{k}\right) / J\left(\mathbf{Q}_{k}\right)$. Therefore, the optimal bit allocation is given by

$$
b_{k}=b+0.5 \log _{2}\left(\sigma_{x_{k}}^{2}\right)-0.5 \sum_{i=0}^{M-1} \frac{\log _{2} \sigma_{x_{i}}^{2}}{J\left(\mathbf{Q}_{i}\right) / J\left(\mathbf{P}_{i}\right)}
$$

Substituting (6.19) into (6.10), we see that all $\sigma_{q_{k}}^{2}$ are equal. For the case of fixed-point quantization, this implies that the rightmost bit (least significant bit) of all channels should be aligned [22, appendix C], [37]. The minimized error variance is $\sigma_{q, \mathrm{opt}}^{2}=c 2^{-2 b} \prod_{k=0}^{M-1}\left(\sigma_{\boldsymbol{x}_{k}}^{2}\right)^{J\left(\mathbf{P}_{k}\right) / J\left(\mathbf{Q}_{k}\right)}$. The coding gain over direct quantization is $G_{\text {opt }}=$ $\sigma_{x}^{2} /\left[\prod_{k=0}^{M-1}\left(\sigma_{x_{k}}^{2}\right)^{J\left(\mathbf{P}_{k}\right) / J\left(\mathbf{Q}_{k}\right)}\right]$. It can be shown that $G_{\text {opt }} \geq 1$ and the equality holds if and only if $\sigma_{x_{k}}^{2}$ is independent of $k$.

\section{Computation Complexity}

Assume for simplicity that both $x(\mathbf{n})$ and $y(\mathbf{n})$ have finite impulse response (FIR) and have $N$ samples each. Direct convolution of $x(\mathbf{n})$ and $y(\mathbf{n})$ requires $N^{2}$ multiplications. Assume that $N$ is much larger than the number of multipliers 
required to implement the filter bank, so that the computation complexity of analysis filter banks in Figs. 8 and 10 can be neglected. Since each subband signal $x_{k}(\mathbf{m})$ (or $y_{k}(\mathbf{m})$ ) has approximately $N J\left(\mathbf{P}_{k}\right) / J\left(\mathbf{Q}_{k}\right)$ samples, each subband convolution seems to require nearly $\left[N J\left(\mathbf{P}_{k}\right) / J\left(\mathbf{Q}_{k}\right)\right]^{2}$ multiplications. However, note that only the $\mathbf{R}_{k} \mathbf{T}_{k}$-fold decimated version of each subband convolution is computed, so only $\left[N J\left(\mathbf{P}_{k}\right) / J\left(\mathbf{Q}_{k}\right)\right]^{2} / J\left(\mathbf{R}_{k} \mathbf{T}_{k}\right)$ multiplications are required. This is repeated for $J(\mathbf{L})$ times to obtain the whole convolution. Therefore, the total number of required multiplications is

$$
\begin{aligned}
J(\mathbf{L}) \sum_{k=0}^{M-1} \frac{\left[N J\left(\mathbf{P}_{k}\right) / J\left(\mathbf{Q}_{k}\right)\right]^{2}}{J\left(\mathbf{R}_{k} \mathbf{T}_{k}\right)} & =N^{2} \sum_{k=0}^{M-1} \frac{J\left(\mathbf{P}_{k}\right)^{2} J(\mathbf{L})}{J\left(\mathbf{Q}_{k}\right)^{2} J\left(\mathbf{R}_{k} \mathbf{T}_{k}\right)} \\
& =N^{2}
\end{aligned}
$$

since $\mathbf{L}=\mathbf{S}_{k} \mathbf{T}_{k}$ and $\mathbf{P}_{k} \mathbf{S}_{k}=\mathbf{Q}_{k} \mathbf{R}_{k}$. In summary, when the computation in analysis bank implementation is negligible, the subband convolution has the same complexity as direct convolution, while providing coding gain.

\section{THE WAVELET TRANSFORM}

The vector space framework is related to the discrete wavelet transform (DWT) [12], [15], [21], [22, ch. 11] very well. In this section, we will illustrate this only for 1-D DWT. The MD case can be obtained similarly. For a continuous-time signal $x(t)$, its DWT is defined as

$$
x_{\mathrm{DWT}}(k, n)=\int_{-\infty}^{\infty} x(t) \underbrace{a^{-k / 2} h\left(n T-a^{-k} t\right)}_{\phi_{k, n}^{*}(t)} d t .
$$

In the above expression, $k$ and $n$ are integer indices, and $a$ and $T$ are some real constant. The DWT hence maps a continuous-time signal $x(t)$ into a 2-D sequence $x_{\mathrm{DWT}}(k, n)$. The quantities $x_{\mathrm{DWT}}(k, n)$ are also called the wavelet coefficients. Note that $\phi_{k, n}^{*}(t)$ are all dilated and shifted versions of $h^{*}(-t)$.

Suppose we want to reconstruct the signal $x(t)$ from its wavelet coefficients. We can use the following expression

$$
\widehat{x}(t)=\sum_{k} \sum_{n} x_{\mathrm{DWT}}(k, n) \underbrace{a^{-k / 2} f\left(a^{-k} t-n T\right)}_{\eta_{k, n}(t)} .
$$

If $\widehat{x}(t)=x(t)$ for all $x(t)$, we get perfect reconstruction (PR) and the above expression is called the inverse discrete wavelet transform (IDWT). Note that $\eta_{k, n}(t)$ are all dilated and shifted versions of the function $f(t)$, which is called the wavelet function, or mother wavelet.

Define the inner product of two continuous-time signals as

$$
(x(t), y(t)) \triangleq \int_{-\infty}^{\infty} x(t) y^{*}(t) d t
$$

We shall consider only signals with finite energy, i.e., signals in the $L^{2}$ space [30], so that the above inner product always exists. By using (7.3), (7.1) can be written in vector space notations as (2.1) and (7.2) can be written as (2.2). In other words, the above DWT analysis/synthesis scheme is in fact a special case of the vector space framework. Assuming that the set $\left\{\eta_{k, n}(t)\right\}$ spans the $L^{2}$ space and that $\eta_{k, n}(t)$ are linearly independent, the following results can be obtained directly from the discussion of Section II:

1) Any two of the following properties imply the other:

a) the Hermitian image property $h^{*}(-t)=f(t)$ (so that $\phi_{k, n}(t)=\eta_{k, n}(t)$ for all $k$ and $n$ )

b) the orthonormality of $\eta_{k, n}(t)$

$$
\int_{-\infty}^{\infty} \eta_{k, n}(t) \eta_{k^{\prime}, n^{\prime}}^{*}(t) d t=\delta\left(k-k^{\prime}\right) \delta\left(n-n^{\prime}\right)
$$

c) the PR property $\widehat{x}(t)=x(t)$.

2) The PR property is satisfied if and only if

$\int_{-\infty}^{\infty} \phi_{k, n}(t) \eta_{k^{\prime}, n^{\prime}}^{*}(t) d t=\delta\left(k-k^{\prime}\right) \delta\left(n-n^{\prime}\right)$

which is the biorthonormality property.

3) Suppose there exists a pair of DWT and IDWT, i.e., the PR condition is satisfied. We can interchange $h(t)$ and $f(t)$ in (7.1) and (7.2) without affecting the PR property.

4) Suppose there exists a pair of DWT and IDWT so that the PR condition is satisfied. We can switch the roles of (7.1) and (7.2) and obtain a synthesis/analysis transmultiplexing systems. More specifically, given a set of wavelet coefficients $x_{\mathrm{DWT}}(k, n)$, we can synthesize a signal $x(t)$ using (7.2). If we analyze such $x(t)$ using (7.1), we can recover the original coefficients $x_{\text {DWT }}(k, n)$.

5) When the functions $\eta_{k, n}(t)$ satisfy the orthonormality in (7.4), we can compute the inner product of two signals by their wavelet coefficients, i.e.,

$$
\int_{-\infty}^{\infty} x(t) g^{*}(t) d t=\sum_{k} \sum_{n} x_{\mathrm{DWT}}(k, n) g_{\mathrm{DWT}}^{*}(k, n)
$$

which is exactly the Parseval Identity in [15]. The biorthonormal case can be obtained similarly.

6) Letting $x(t)=g(t)$ in (7.6), we get the energy conservation equation. Furthermore, the energy is preserved for all $x(t)$ if and only if the orthonormality condition is satisfied.

\section{Wavelet Convolution Theorem}

We can also derive the "wavelet convolution theorem" for the biorthonormal case (with the orthonormal case covered as a special case). Unlike the filter bank case, it is not easy to derive this starting from the Parseval's relation, because we would need to shift $g(t)$ in (7.6) continuously to obtain the whole convolution. Instead, we will use the graphical derivation as we did in Fig. 11. First, we let $h_{k}(t) \triangleq a^{-k / 2} h\left(a^{-k} t\right)$ and $f_{k}(t) \triangleq a^{-k / 2} f\left(a^{-k} t\right)$. We can then rewrite (7.1) as

$$
x_{\text {DWT }}(k, n)=\int_{-\infty}^{\infty} x(t) h_{k}\left(n a^{k} T-t\right) d t
$$

and (7.2) as

$$
x(t)=\sum_{k} \sum_{n} x_{\mathrm{DWT}}(k, n) f_{k}\left(t-n a^{k} T\right)
$$




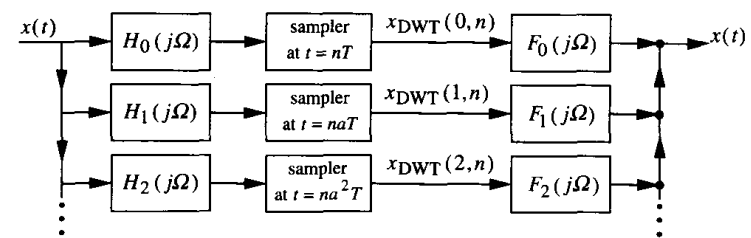

Fig. 13. Filter bank implementation of a DWT/IDWT system with input $x(t)$. The signals $x_{\mathrm{DWT}}(k, n)$ in this figure indicate continuous-time impulse trains.

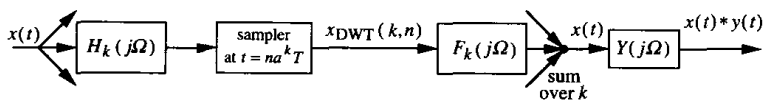

(a)

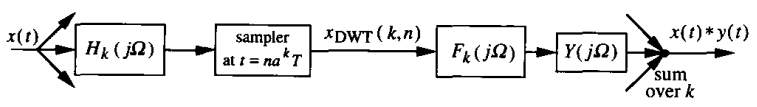

(b)

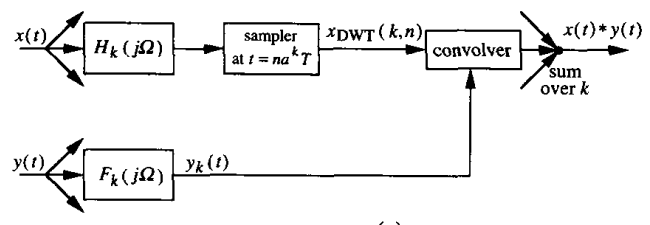

(c)

Fig. 14. DWT subband convolution of $x(t)$ and $y(t)$.

since $\widehat{x}(t)=x(t)$ for the biorthonormal case. These two equations give the filter bank implementation of a DWT/IDWT system shown in Fig. 13. In this figure, $H_{k}(j \Omega)$ and $F_{k}(j \Omega)$ denote the Fourier transform of $h_{k}(t)$ and $f_{k}(t)$, respectively. The sampler in the $k$ th channel samples the input signal at $t=n a^{k} T$ and produces an impulse train at the output. It can be verified that the output is $\sum_{n} x_{\mathrm{DWT}}(k, n) \delta\left(t-n a^{k} T\right)$, where $\delta(t)$ denotes the continuous-time impulse function.

If we want to compute the convolution of two continuoustime signals $x(t)$ and $y(t)$, we can use the system in Fig. 14(a), where $Y(j \Omega)$ is the Fourier transform of $y(t)$. Note that only one channel in the filter bank of Fig. 13 is shown. With $Y(j \Omega)$ moved to the left, Fig. 14(a) can be redrawn as Fig. 14(b). It can be verified that Fig. 14(b) is equivalent to Fig. 14(c), which gives the following theorem:

Theorem 10: Wavelet convolution theorem (biorthonormal case): Suppose there exists a pair of DWT and IDWT so that the PR condition (biorthonormality) is satisfied. The convolution of two signals $x(t)$ and $y(t)$ can be computed as

$$
\begin{aligned}
x(t) * y(t) & \triangleq \int_{-\infty}^{\infty} x(\tau) y(t-\tau) d \tau \\
& =\sum_{k} x_{\mathrm{DWT}}(k, n) y_{k}\left(t-n a^{k} T\right)
\end{aligned}
$$

where $x_{\mathrm{DWT}}(k, n)$ are the wavelet coefficients of $x(t)$, and $y_{k}(t)$ is the output of the filter $f_{k}(t)$ with the input $y(t)$.

Remarks: If we let let $y(t)=\delta(t)$ in (7.9) so that $y_{k}(t)=$ $f_{k}(t),(7.9)$ is then reduced to (7.8) nicely.

\section{REMARKS AND CONCLUSION}

Here, we add a remark on the orthonormality defined in (3.15) and the biorthonormality in (3.18). For the 1-D case with all $P_{k}=1,(3.15)$ becomes

$$
\sum_{n=-\infty}^{\infty} f_{k}\left(n-Q_{k} m\right) f_{k^{\prime}}^{*}\left(n-Q_{k^{\prime}} m^{\prime}\right)=\delta\left(k-k^{\prime}\right) \delta\left(m-m^{\prime}\right)
$$

for all $k, k^{\prime}=0, \cdots, M-1$ and all $m$ and $m^{\prime}$. In [38], it has been shown that this condition can be simplified to

$$
\sum_{n=-\infty}^{\infty} f_{k}(n) f_{k^{\prime}}^{*}\left(n-\operatorname{gcd}\left(Q_{k}, Q_{k^{\prime}}\right) i\right)=\delta\left(k-k^{\prime}\right) \delta(i)
$$

for all $k, k^{\prime}=0, \cdots, M-1$ and all $i$, where $\operatorname{gcd}\left(Q_{k}, Q_{k^{\prime}}\right)$ denotes the greatest common divisor of $Q_{k}$ and $Q_{k^{\prime}}$. For the MD case, we can show that the orthonormality condition in (3.15) can be rewritten in terms of $f_{k, \mathbf{p}}(\mathbf{n})$, the $\mathbf{P}_{k}$-fold polyphase components of $f_{k}(\mathbf{n})$, as follows

$$
\begin{aligned}
\sum_{\mathbf{n} \in \mathcal{N}} f_{k, \mathbf{p}}\left(\mathbf{n}+\mathbf{A}_{k} \mathbf{p}\right) f_{k^{\prime}, \mathbf{p}^{\prime}}^{*}(\mathbf{n} & \left.+\mathbf{A}_{k^{\prime}} \mathbf{p}^{\prime}-\operatorname{gcld}\left(\mathbf{S}_{k}, \mathbf{S}_{k^{\prime}}\right) \mathbf{i}\right) \\
& =\delta\left(k-k^{\prime}\right) \delta\left(\mathbf{p}-\mathbf{p}^{\prime}\right) \delta(\mathbf{i})
\end{aligned}
$$

for all $k, k^{\prime}=0, \cdots, M-1, \mathbf{p} \in \mathcal{N}\left(\mathbf{P}_{k}\right), \mathbf{p}^{\prime} \in \mathcal{N}\left(\mathbf{P}_{k^{\prime}}\right)$, and all $\mathbf{i} \in \mathcal{N}$, where $\operatorname{gcld}\left(\mathbf{Q}_{k}, \mathbf{Q}_{k^{\prime}}\right)$ denotes the greatest common left divisor [31] of $\mathbf{Q}_{k}$ and $\mathbf{Q}_{k^{\prime}}$. For the case where all $\mathbf{P}_{k}=\mathbf{I}$, this can be simplified further as

$$
\sum_{\mathbf{n} \in \mathcal{N}} f_{k}(\mathbf{n}) f_{k^{\prime}}^{*}\left(\mathbf{n}-\operatorname{gcld}\left(\mathbf{Q}_{k}, \mathbf{Q}_{k^{\prime}}\right) \mathbf{i}\right)=\delta\left(k-k^{\prime}\right) \delta(\mathbf{i}) .
$$

Similarly, the biorthonormality in (3.18) can also be simplified. Note that although the above simplifies the expression of orthonormality, it does not necessarily simplify derivations of results in this paper.

In this paper, we proposed the vector space notations which provide a framework for deriving a number of theoretical results in maximally decimated filter banks. Because of the generality of vector space, these theoretical results can be applied to the most general filter banks, namely, MD nonuniform rational filter banks. We have shown the relation among the Hermitian image property, orthonormality, and the perfect reconstruction property in a maximally decimated filter bank. With vector space notations, it can also been shown that perfect reconstruction is achieved if and only if the biorthonormality condition is satisfied. We proved that we can obtain a PR synthesis/analysis transmultiplexer from a PR maximally decimated analysis/synthesis filter bank, and vice versa. We also derived the MD subband convolution scheme starting from the generalized Parseval's relation. Put in vector space notations, many of these results become very explicit and easy to prove. Therefore, the vector space interpretation provides a very powerful tool in deriving these filter bank theoretical results. To illustrate the generality of this framework, we have also applied it to another analysis/synthesis scheme, namely, the wavelet transform, and obtained a number of useful results. In particular, we have derived the so-çalled wavelet convolution theorem. 


\section{APPENDIX A}

We need to prove

$$
\begin{aligned}
h_{k}^{*}\left(\mathbf{Q}_{k} \mathbf{m}-\mathbf{P}_{k} \mathbf{n}\right) & =f_{k}\left(\mathbf{P}_{k} \mathbf{n}-\mathbf{Q}_{k} \mathbf{m}\right) \text { for } \mathbf{m} \in \mathcal{N} \\
& \Longleftrightarrow h_{k}^{*}(\mathbf{n})=f_{k}(-\mathbf{n})
\end{aligned}
$$

where $\mathbf{n} \in \mathcal{N}$ is the space domain index. It is clear the right-hand side implies the left-hand side of this statement. Conversely, suppose $h_{k}^{*}\left(\mathbf{Q}_{k} \mathbf{m}-\mathbf{P}_{k} \mathbf{n}\right)=f_{k}\left(\mathbf{P}_{k} \mathbf{n}-\mathbf{Q}_{k} \mathbf{m}\right)$, we want to show $h_{k}^{*}(\mathbf{n})=f_{k}(-\mathbf{n})$ for all $\mathbf{n}$. Because $\mathbf{P}_{k}$ and $\mathbf{Q}_{k}$ are left coprime, there exist integer matrices $\mathbf{A}$ and $\mathbf{B}$ such that $\mathbf{Q}_{k} \mathbf{B}+\mathbf{P}_{k} \mathbf{A}=\mathbf{I}$ (generalized Bezout theorem [31]-[33]. So, any $p \in \mathcal{N}$ can be expressed as

$$
\mathbf{p}=\mathbf{Q}_{k} \underbrace{\mathbf{A p}}_{\mathbf{m}}+\mathbf{P}_{k} \underbrace{\mathbf{B p}}_{-\mathbf{n}} .
$$

Therefore, $h_{k}^{*}(\mathbf{p})=h_{k}^{*}\left(\mathbf{Q}_{k} \mathbf{m}-\mathbf{P}_{k} \mathbf{n}\right)=f_{k}\left(\mathbf{P}_{k} \mathbf{n}-\mathbf{Q}_{k} \mathbf{m}\right)=$ $f_{k}(-\mathbf{p})$ for all $\mathbf{p} \in \mathcal{N}$.

\section{APPENDIX B}

Any nonsingular rational matrix $\mathbf{H}$ can be written as $\mathbf{H}=$ $\mathbf{Q}^{-1} \mathbf{P}$, where $\mathbf{P}$ and $\mathbf{Q}$ are left coprime integer matrices. This is called an irreducible left matrix fraction description (MFD) of $\mathbf{H}$ [32], [33]. Similarly, $\mathbf{H}$ can be written as $\mathbf{H}=\mathbf{R S}^{-1}$ with $\mathbf{R}$ and $\mathbf{S}$ being right coprime integer matrices. This is called an irreducible right MFD of $\mathbf{H}$. We then have $\mathbf{P S}=\mathbf{Q R}$. It has been shown that the denominator matrices of all irreducible right or left MFD's have the same absolute determinant, i.e., $J(\mathbf{Q})=J(\mathbf{S})$ [34]. With these properties, we can prove the following lemma:

Lemma 1: Given $\mathbf{P}$ and $\mathbf{Q}$ (both nonsingular), we first find $\mathbf{R}$ and $\mathbf{S}$ which are right coprime such that $\mathbf{P S}=\mathbf{Q R}$. In other words, we find $\mathbf{R S}^{-1}$ which is a irreducible right MFD of $\mathbf{Q}^{-1} \mathbf{P}$. Then

$$
\mathbf{P r}=\mathbf{Q m} \quad \text { if and only if } \begin{cases}\mathbf{r} & =\mathbf{S} \mathbf{j} \in \text { LAT }(\mathbf{S}), \text { and } \\ \mathbf{m} & =\mathbf{R j} \in \text { LAT }(\mathbf{R})\end{cases}
$$

Proof: Suppose $\mathbf{k}=\mathbf{P r}=\mathbf{Q m}$, so $\mathbf{k} \in \operatorname{LAT}(\mathbf{P}) \cap$ LAT $(Q)$. It can be proved that the intersection of two lattices is a lattice generated by the $1 \mathrm{crm}$ of the two generating matrices [31], [34], i.e., $\operatorname{LAT}(\mathbf{P}) \cap \operatorname{LAT}(\mathbf{Q})=\operatorname{LAT}(\operatorname{lcrm}(\mathbf{P}, \mathbf{Q}))$. Hence we have $\mathbf{k} \in \operatorname{LAT}(\mathbf{P}) \cap \operatorname{LAT}(\mathbf{Q})=\operatorname{LAT}(\operatorname{lcm}(\mathbf{P}, \mathbf{Q}))$, so $\mathbf{k}=\operatorname{lcrm}(\mathbf{P}, \mathbf{Q}) \mathbf{j}$. It has been shown that if $\mathbf{R}$ and $\mathbf{S}$ are right coprime, then $\mathbf{P S}=\mathbf{Q R}$ is indeed an $\operatorname{lcrm}(\mathbf{P}, \mathbf{Q})$ [34]. Therefore, $\mathbf{k}=\mathbf{P S} \mathbf{j}=\mathbf{Q R \mathbf { j }}$ and hence $\mathbf{r}=\mathbf{S j}$ and $\mathbf{m}=\mathbf{R j}$. The proof of the converse is straightforward.

$\triangle \triangle \triangle$

We can generalize Lemma 1 to the following lemma:

Lemma 2: Given nonsingular left coprime matrices $\mathbf{P}$ and $\mathbf{Q}$, we first find the corresponding $\mathbf{R}$ and $\mathbf{S}$ as in Lemma 1 . Furthermore, since $\mathbf{P}$ and $\mathbf{Q}$ are left coprime, we can find $\mathbf{A}$ and $\mathbf{B}$ such that $\mathbf{P A}+\mathbf{Q B}=\mathbf{I}$. Then,

$$
\begin{aligned}
& \mathbf{P r}+\mathbf{p}=\mathbf{Q m} \quad \text { if and only if } \\
& \mathbf{r}+\mathbf{A p}=\mathbf{S j}, \text { i.e., } \mathbf{r}+\mathbf{A p} \in \operatorname{LAT}(\mathbf{S}) .
\end{aligned}
$$

Proof: Suppose $\mathbf{P r}+\mathbf{p}=\mathbf{Q m}$. Using $\mathbf{P A}+\mathbf{Q B}=\mathbf{I}$, we have $\mathbf{P A p}+\mathbf{Q B p}=\mathbf{p}$ so that $\mathbf{P r}+\mathbf{P A p}+\mathbf{Q B p}=\mathbf{Q m}$. Rearrange terms to get $\mathbf{P}(\mathbf{r}+\mathbf{A p})=\mathbf{Q}(\mathbf{m}-\mathbf{B p})$. Using Lemma 1, we conclude $\mathbf{r}+\mathbf{A p}=\mathbf{S j} \in \operatorname{LAT}(\mathbf{S})$. Conversely, suppose $\mathbf{r}+\mathbf{A p}=\mathbf{S} \mathbf{j}$, or $\mathbf{r}=\mathbf{S} \mathbf{j}-\mathbf{A p}$. Compute $\mathbf{P r}$ as follows: $\mathbf{P r}=\mathbf{P S j}-\mathbf{P A p}=\mathbf{P S} \mathbf{j}-\mathbf{p}+\mathbf{Q B p}=$ $\mathbf{Q} \underbrace{(\mathbf{R j}+\mathbf{B p})}-\mathbf{p}$. So, Pr $+\mathbf{p}=\mathbf{Q m} . \quad \triangle \triangle \triangle$ m

\section{REFERENCES}

[1] P. P. Vaidyanathan, "Theory and design of $M$-channel maximally decimated quadrature mirror filters with arbitrary $M$, having the perfectreconstruction property," IEEE Trans. Acoust., Speech, Signal Processing, vol. 35, no. 4, pp. 476-492, Apr. 1987.

[2] M. Vetterli, "Filter banks allowing for perfect reconstruction," Signal Processing, vol. 10, pp. 219-244, Apr. 1986

[3] M. J. T. Smith and T. P. Barnwell III, "A new filter-bank theory for time-frequency representation," IEEE Trans. Acoust., Speech, Signal Processing, vol. 35, pp. 314-327, Mar. 1987.

[4] P.-Q. Hoang and P. P. Vaidyanathan, "Non-uniform multirate filter banks: Theory and design," in Proc. IEEE Int. Symp. Circuits, Systems (Portland), 1989.

[5] K. Nayebi, T. P. Barnwell III, and M. J. T. Smith, "The design of perfect reconstruction nonuniform band filter banks," in Proc. IEEE Int. Conf. Acoust., Speech, Signal Processing, (Canada), 1991, pp. 1781-1784,

[6] J. Kovačević and M. Vetterli, "Perfect reconstruction filter banks with rational sampling rate changes," in Proc. IEEE Int. Conf. Acoust., Speech, Signal Processing (Canada), 1991, pp. 1785-1788.

[7] R. A. Gopinath and C. S. Burrus, "On upsampling, downsampling and rational sampling rate filter banks," IEEE Trans. Signal Processing, Apr. 1994.

[8] B. Paillard, J. Soumagne, P. Mabilleau, and S. Morissette, "Filters for subband coding, analytical approach," in Proc. IEEE Int. Conf. Acoustics, Speech, Signal Processing (Dallas, TX), Apr. 1987, pp. 50.2.1-50.2.5

[9] E. H. Adelson, E. P. Simoncelli, and R. Hingorani, "Orthogonal pyramid transforms for image coding," in Proc. SPIE Visual Commun., Image Processing, 1987, pp. 50-58.

[10] M. Vetterli and D. Le Gall, "Perfect reconstruction FIR filter banks: Some properties and factorization," IEEE Trans. Acoust., Speech, Signal Processing, vol. 37, no. 7, pp. 1057-1071, July 1989.

[11] K. Nayebi, T. P. Barnwell III, and M. J. T. Smith, "Time domain conditions for exact reconstruction in analysis/synthesis systems based on maximally decimated filter banks," Proc. Southeastern Symp. Syst. Theory, Mar. 1987.

[12] A. N. Akansu and R. A. Haddad, Multiresolution Signal Decomposition Transforms, Subbands, and Wavelets. New York: Academic, 1992.

[13] H. S. Malvar, Signal Processing with Lapped Transforms. Norwood, MA: Artech, 1992.

[14] V. Nuri and R. H. Bamberger, "A theoretical framework for the analysis and design of size-limited multirate filter banks," in Proc. IEEE-SP Int. Symp. Time-Frequency, Time-Scale Anal., 1992.

[15] C. K. Chui, An Introduction to Wavelets. New York: Academic, 1992.

[16] M. Vetterli and C. Herley, "Wavelets and filter banks: theory and design," IEEE Trans. Signal Processing, vol. 40, no. 9, pp. 2209-2232, Sept. 1992.

[17] IEEE Trans. Commun., Special Issue on Transmultiplexers, vol. 30, no. 7, July 1982.

[18] M. Vetterli, "Perfect transmultiplexers," in Proc. IEEE Int. Conf. Acoust., Speech, Signal Processing (Tokyo, Japan), Apr. 1986, pp. 2567-2570.

[19] R. D. Koilpillai, T. Q. Nguyen, and P. P. Vaidyanathan, "Some results in the theory of crosstalk-free transmultiplexers," IEEE Trans. Signal Processing, vol. 39, no. 10, pp. 2174-2183, Oct. 1991.

[20] P. P. Vaidyanathan, "Orthonormal and biorthonormal filter-banks as convolvers, and convolutional coding gain," IEEE Trans. Signal Processing, vol. 41, no. 6, pp. 2110-2130, June 1993 .

[21] I. Daubechies, Ten Lectures on Wavelets, SIAM, CBMS series, Apr 1992.

[22] P. P. Vaidyanathan, Multirate Systems and Filter Banks. Englewood Cliffs, NJ: Prentice-Hall, 1993.

[23] D. E. Dudgeon and R. M. Mersereau, Multidimensional Digital Signal Processing. Englewood Cliffs, NJ: Prentice-Hall, 1984.

[24] R. Ansari and C.-L. Lau, "Two-dimensional IIR filters for exact reconstruction in tree structured sub-band decomposition," Electron. Lett., vol. 23, pp. 633-634, June 1987. 
[25] E. Viscito and J. P. Allebach, "Design of perfect reconstruction multidimensional filter banks using cascaded Smith form matrices," in Proc. IEEE Int. Symp. Circuits, Systems (Espoo, Finland), June 1988, pp. 831-834.

[26] G. Karlsson and M. Vetterli, "Theory of two-dimensional multirate filter banks," IEEE Trans. Acoust., Speech, Signal Processing, vol. 38, no. 6, pp. 925-937, June 1990.

[27] P. P. Vaidyanathan, "Fundamentals of multidimensional multirate digital signal processing," Sadhana, vol. 15, pp. 157-176, Nov. 1990.

[28] E. Viscito and J. P. Allebach, "The analysis and design of multidimensional FIR perfect reconstruction filter banks for arbitrary sampling lattices," IEEE Trans. Circuits, Syst., vol. 38, no. 1, pp. 29-41, Jan. 1991.

[29] J. W. Woods, Subband Image Coding. Norwood, MA: Kluwer, 1991.

[30] D. G. Luenberger, Optimization by Vector Space Methods. New York: Wiley, 1969.

[31] C. C. MacDuffee, The Theory of Matrices. New York: Chelsea, 1946.

[32] T. Kailath, Linear Systems. Englewood Cliffs, NJ: Prentice-Hall, 1980.

[33] M. Vidyasagar, Control System Synthesis: A Factorization Approach. Cambridge, MA: The M.I.T. Press, 1985.

[34] T. Chen and P. P. Vaidyanathan, "The role of integer matrices in multidimensional multirate systems," IEEE Trans. Signal Processing, vol. 41, no. 3, pp. 1035-1047, Mar. 1993.

[35] N. S. Jayant and P. Noll, Digital Coding of Waveforms. Englewood Cliffs, NJ: Prentice-Hall, 1984.

[36] V. Sathe and P. P. Vaidyanathan, "Effects of multirate systems on the statistical properties of random inputs," IEEE Trans. Signal Processing, vol. 41, no. 1, pp. 131-146, Jan. 1993.

[37] A. K. Soman and P. P. Vaidyanathan, "Coding gain in paraunitary analysis/synthesis systems," IEEE Trans. Signal Processing, vol. 41, no. 5, pp. 1824-1835, May 1993.

[38] A. K. Soman and P. P. Vaidyanathan, "Paraunitary filter banks and wavelet packets," in Proc. IEEE Int. Conf. Acoust, Speech, Signal Processing (San Francisco, CA), vol. 4, Mar. 1992, pp. 397-400.

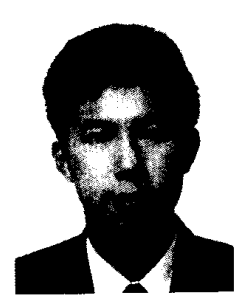

Tsuhan Chen (S'90-M'93) was born in Taipei, Taiwan, R.O.C., on November 7, 1965. He received the B.S. degree from the National Taiwan University in 1987 and the M.S. and Ph.D. degrees from the California Institute of Technology, Pasadena, in 1990 and 1993, respectively, all in electrical engineering.

From January 1993 and July 1993, he was a part-time Member of the Technical Staff at the Jet Propulsion Laboratory, Pasadena, CA. He was a Postdoctoral Research Fellow at the California Institute of Technology from July 1993 to August 1993. In August 1993 , he joined AT\&T Bell Laboratories, Holmdel, NJ, as a Member of the Technical Staff. His current research interests include image/video coding and multidimensional multirate signal processing.

Dr. Chen is a member of the Phi Tau Phi Scholastic Honor Society. He received the Charles Wilts Prize for outstanding independent research in electrical engineering, leading to the Ph.D. degree.

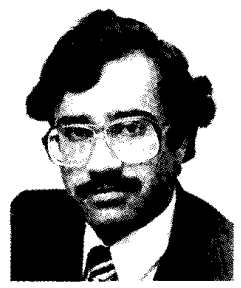

P. P. Vaidyanathan (S'80-M'83-SM'88-F'91) was born in Calcutta, India, on October 16, 1954 $\mathrm{He}$ received the B.Sc. (Hons.) degree in physics and the B.Tech. and M.Tech. degrees in radiophysics and electronics, all from the University of Calcutta, India, in 1974, 1977, and 1979, respectively, and the Ph.D. degree in electrical and computer engineering from the University of California at Santa Barbara in 1982.

He was a postdoctoral fellow at the University of California, Santa Barbara, from September 1982 to March 1983. In March 1983, he joined the Electrical Engineering Department of the California Institute of Technology, Pasadena, as an Assistant Professor, and since 1993 has been Professor of Electrical Engineering. He has authored a number of papers in IEEE journals, including a paper on linear-phase perfect reconstruction filter banks in the IEEE TRansactions on Signal Processing, is the author of Multirate Systems and Filter Banks, and has authored several chapters for various signal processing handbooks. His main research interests are in digital signal processing, multirate systems, wavelet transforms, and adaptive filtering.

Dr. Vaidyanathan was a recipient of the Award for Excellence in Teaching at the California Institute of Technology, for 1983-1984 and 1992-1993. He also received the NSF's Presidential Young Investigator Award in 1986. In 1989, he received the IEEE ASSP Senior Award for his paper on multirate perfect-reconstruction filter banks. In 1990, he was recepient of the S. K. Mitra Memorial Award from the Institute of Electronics and Telecommunications Engineers, India, for his joint paper in the IETE journal. He served as Vice Chairman of the Technical Program Committee for the 1983 IEEE International Symposium on Circuits and Systems and as the Technical Program Chairman for the 1992 IEEE International Symposium on Circuits and Systems. He was an Associate Editor for the IEEE TRAnSACTIONS ON CrRCUITS AND SYSTEMS for 1985-1987 and is currently an Associate Editor for IEEE Signal Processing LetTers and a Consulting Editor for Applied and Computational Harmonic Analysis. 